\title{
COMPUTING NONCOMMUTATIVE DEFORMATIONS OF PRESHEAVES AND SHEAVES OF MODULES
}

\author{
EIVIND ERIKSEN
}

\begin{abstract}
We describe a noncommutative deformation theory for presheaves and sheaves of modules that generalizes the commutative deformation theory of these global algebraic structures, and the noncommutative deformation theory of modules over algebras due to Laudal.

In the first part of the paper, we describe a noncommutative deformation functor for presheaves of modules on a small category, and an obstruction theory for this functor in terms of global Hochschild cohomology. An important feature of this obstruction theory is that it can be computed in concrete terms in many interesting cases.

In the last part of the paper, we describe noncommutative deformation functors for sheaves and quasi-coherent sheaves of modules on a ringed space $(X, \mathcal{A})$. We show that for any good $\mathcal{A}$-affine open cover $\mathrm{U}$ of $X$, the forgetful functor $\mathrm{QCoh}(\mathcal{A}) \rightarrow \operatorname{PreSh}(\mathrm{U}, \mathcal{A})$ induces an isomorphism of noncommutative deformation functors.

Applications. We consider noncommutative deformations of quasi-coherent $\mathcal{A}$-modules on $X$ when $(X, \mathcal{A})=\left(X, \mathcal{O}_{X}\right)$ is a scheme or $(X, \mathcal{A})=(X, \mathcal{D})$ is a D-scheme in the sense of Beilinson and Bernstein. In these cases, we may use any open affine cover of $X$ closed under finite intersections to compute noncommutative deformations in concrete terms using presheaf methods. We compute the noncommutative deformations of the left $\mathcal{D}_{X}$-module $\mathcal{O}_{X}$ when $X$ is an elliptic curve as an example.
\end{abstract}

\section{INTRODUCTION}

Deformation theory was formalized by Grothendieck in the language of schemes in the 1950s, and is described in the series of Bourbaki seminar expositions Fondements de la géométrie algébrique [9]; see in particular Grothendieck [10, 11. The general philosophy is best described by the following quotation:

La méthode générale consiste toujours à faire des constructions formelles, ce qui consiste essentiellement à faire de la géométrie algébrique sur un anneau artinien, et à en tirer des conclusions de nature "algébrique" en utilisant les trois théorèmes fondamentaux (Grothendieck [10], p. 11)

We shall follow Grothendieck's philosophy closely, and we are therefore led to the study of functors of (noncommutative) Artin rings.

Let $k$ be an algebraically closed field, and let I denote the category of local Artinian commutative $k$-algebras with residue field $k$, with local homomorphisms. A functor of Artin rings is a covariant functor D : I $\rightarrow$ Sets such that $\mathrm{D}(k)$ only contains one element. In Schlessinger [16], criteria for functors of Artin rings to

This research has been supported by a postdoctoral grant awarded by the Norwegian Research Council, project no. 157740/432, and a grant awarded by the Mittag-Leffler Institute. 
have a pro-representable hull, respectively for functors of Artin rings to be prorepresentable, were given.

Let $\mathrm{A}$ be an Abelian $k$-category, and let $X$ be an object of A. The flat deformation functor $\operatorname{Def}_{X}: I \rightarrow$ Sets of $X$ in A is a functor of Artin rings. In many cases, it has a pro-representing hull $\mathrm{H}\left(\operatorname{Def}_{X}\right)$, see Schlessinger [16, Laudal [15, and there are constructive methods for finding $\mathrm{H}\left(\operatorname{Def}_{X}\right)$, see Laudal [15, [13. In fact, if there exists an obstruction theory for $\operatorname{Def}_{X}$ with finite dimensional cohomology $\mathrm{H}^{n}(X)$ for $n=1,2$, then $\operatorname{Def}_{X}$ has a pro-representing hull, given algorithmically in terms of the vector spaces $\mathrm{H}^{n}(X)$ for $n=1,2$ and certain generalized symmetric Massey products on them.

When $\mathrm{A}=\operatorname{Mod}(A)$, the category of left modules over an associative $k$-algebra $A$, Laudal introduced a generalization of the deformation functor $\operatorname{Def}_{M}: \mathrm{I} \rightarrow$ Sets for a left $A$-module $M$ in Laudal [14. He considered the category a ${ }_{p}$ of $p$-pointed Artinian rings for any integer $p \geq 1$, and constructed a noncommutative deformation functor Def $_{\mathcal{M}}: \mathrm{a}_{p} \rightarrow$ Sets for any finite family $\mathcal{M}=\left\{M_{1}, \ldots, M_{p}\right\}$ of left $A$-modules. This deformation functor has an obstruction theory with cohomology $\left(\operatorname{Ext}_{A}^{n}\left(M_{j}, M_{i}\right)\right)$, and a pro-representing hull $\mathrm{H}\left(\operatorname{Def}_{\mathcal{M}}\right)$, given algorithmically in terms of the vector spaces $\left(\operatorname{Ext}_{A}^{n}\left(M_{j}, M_{i}\right)\right)$ and certain generalized Massey products on them.

The objects in the category $\mathrm{a}_{p}$ are Artinian rings $R$, together with ring homomomorphisms $k^{p} \rightarrow R \rightarrow k^{p}$ such that the composition is the identity, and such that $R$ is $I$-adic complete for $I=\operatorname{ker}\left(R \rightarrow k^{p}\right)$. The morphisms are the natural commutative diagrams. In section 1, we give a systematic introduction to functors $\mathrm{D}: \mathrm{a}_{p} \rightarrow$ Sets of noncommutative Artian rings, following Laudal [14].

The idea is that there is a noncommutative deformation functor $\operatorname{Def}_{X}: \mathrm{a}_{p} \rightarrow$ Sets for any finite family $X=\left\{X_{1}, \ldots, X_{p}\right\}$ of algebraic or algebro-geometric objects. The restrictions of Def $X_{X}$ along the $p$ natural full embeddings of categories $\mathrm{a}_{1} \subseteq \mathrm{a}_{p}$ are the noncommutative deformation functors $\operatorname{Def}_{X_{i}}: a_{1} \rightarrow$ Sets, and the restriction of $\operatorname{Def}_{X_{i}}$ to $I \subseteq \mathrm{a}_{1}$ is the commutative deformation functor $\operatorname{Def}_{X_{i}}^{c}: I \rightarrow$ Sets. When these deformation functors have pro-representing hulls, we show that

$$
\mathrm{H}\left(\operatorname{Def}_{X}\right)^{\mathrm{comm}} \cong \underset{1 \leq i \leq p}{\oplus} \mathrm{H}\left(\operatorname{Def}_{X_{i}}\right)^{\mathrm{comm}} \cong \underset{1 \leq i \leq p}{\oplus} \mathrm{H}\left(\operatorname{Def}_{X_{i}}^{c}\right)
$$

We remark that the hull $\mathrm{H}\left(\operatorname{Def}_{X}\right)$ is not isomorphic to $\oplus_{i} \mathrm{H}\left(\operatorname{Def}_{X_{i}}\right)$ in general. We also remark that the noncommutative deformation functor $\operatorname{Def}_{X}: a_{p} \rightarrow$ Sets of a family $X=\left\{X_{1}, \ldots, X_{p}\right\}$ is not the same as the noncommutative deformation functor $\operatorname{Def}_{X}: \mathrm{a}_{1} \rightarrow$ Sets of the direct sum $X=X_{1} \oplus \cdots \oplus X_{p}$.

Let $\operatorname{PreSh}(\mathrm{c}, \mathcal{A})$ be the category of presheaves of left $\mathcal{A}$-modules on $\mathrm{c}$, where $\mathrm{c}$ is a small category and $\mathcal{A}$ is a $k$-algebra of presheaves on c. We consider an Abelian $k$-category A such that $\mathrm{A} \subseteq \operatorname{PreSh}(\mathrm{c}, \mathcal{A})$ is a full subcategory, and construct a noncommutative deformation functor $\operatorname{Def}_{\mathcal{F}}^{A}: a_{p} \rightarrow$ Sets for any finite family $\mathcal{F}=\left\{\mathcal{F}_{1}, \ldots, \mathcal{F}_{p}\right\}$ of objects in $\mathrm{A}$ in section 2. We remark that we use a notion of matric freeness, introduced by Laudal, to replace flatness in the definition of the deformation functor. It is not clear if these notions are equivalent if $p \geq 2$.

In section 3 - 5, we consider deformations in the category $\mathrm{A}=\operatorname{PreSh}(\mathrm{c}, \mathcal{A})$ of presheaves. We describe the noncommutative deformation functor $\operatorname{Def}_{\mathcal{F}}: \mathrm{a}_{p} \rightarrow$ Sets of any finite family $\mathcal{F}=\left\{\mathcal{F}_{1}, \ldots, \mathcal{F}_{p}\right\}$ of presheaves of left $\mathcal{A}$-modules on $\mathrm{c}$ in concrete terms in section 3, and use this to develop an obstruction theory for $\operatorname{Def}_{\mathcal{F}}$ with cohomology $\left(\mathrm{HH}^{n}\left(\mathrm{c}, \mathcal{A}, \mathcal{H} \operatorname{Hom}_{k}\left(\mathcal{F}_{j}, \mathcal{F}_{i}\right)\right)\right)$ in section 5 . The global Hochschild 
cohomology $\mathrm{HH}^{n}\left(\mathrm{c}, \mathcal{A}, \mathcal{H o m}_{k}\left(\mathcal{F}_{j}, \mathcal{F}_{i}\right)\right)$ of $\mathcal{A}$ with values in $\mathcal{H o m}_{k}\left(\mathcal{F}_{j}, \mathcal{F}_{i}\right)$ on c is described in detail in section 4

Theorem 1. Let c be a small category, let $\mathcal{A}$ be a presheaf of $k$-algebras on $\mathrm{c}$, and let $\mathcal{F}=\left\{\mathcal{F}_{1}, \ldots, \mathcal{F}_{p}\right\}$ be a finite family of presheaves of left $\mathcal{A}$-modules on c. If $\operatorname{dim}_{k} \mathrm{HH}^{n}\left(\mathrm{c}, \mathcal{A}, \mathcal{H o m}_{k}\left(\mathcal{F}_{j}, \mathcal{F}_{i}\right)\right)<\infty$ for $1 \leq i, j \leq p, n=1,2$, then the noncommutative deformation functor $\operatorname{Def}_{\mathcal{F}}: a_{p} \rightarrow$ Sets of $\mathcal{F}$ in $\operatorname{PreSh}(\mathrm{c}, \mathcal{A})$ has a pro-representing hull $\mathrm{H}\left(\operatorname{Def}_{\mathcal{F}}\right)$, completely determined by the $k$-linear spaces $\mathrm{HH}^{n}\left(\mathrm{c}, \mathcal{A}, \mathcal{H}_{\mathrm{om}}\left(\mathcal{F}_{j}, \mathcal{F}_{i}\right)\right)$ for $1 \leq i, j \leq p, n=1,2$, together with some generalized Massey products on them.

In section [6] we consider deformations in the category $\mathrm{A}=\operatorname{Sh}(X, \mathcal{A})$ of sheaves of left $\mathcal{A}$-modules on $X$, where $(X, \mathcal{A})$ is a ringed space over $k$. We may consider $\operatorname{Sh}(X, \mathcal{A})$ as a full subcategory of $\operatorname{PreSh}(\mathrm{c}(X), \mathcal{A})$, where $\mathrm{c}(X)$ is the category with open subsets $U \subseteq X$ as objects, and opposite inclusion $U \supseteq V$ as morphisms. Moreover, $\operatorname{Sh}(X, \mathcal{A})$ is an Abelian $k$-category. We show that for any finite family $\mathcal{F}$ in $\operatorname{Sh}(X, \mathcal{A})$, the natural forgetful functor $\operatorname{Sh}(X, \mathcal{A}) \rightarrow \operatorname{PreSh}(\mathrm{c}(X), \mathcal{A})$ induces an isomorphism $\operatorname{Def}_{\mathcal{F}}^{s h} \rightarrow \operatorname{Def}_{\mathcal{F}}$ of deformation functors. However, this result is not very useful for computational purposes, since the category $\mathrm{c}(X)$ consisting of all open sets in $X$ is usually too big to allow for effective computations.

In section 7, we consider deformations in the category $\mathrm{A}=\mathrm{QCoh}(\mathcal{A})$ of quasicoherent sheaves of left $\mathcal{A}$-modules on $X$, where $(X, \mathcal{A})$ is a ringed space over $k$. If $X$ has an $\mathcal{A}$-affine open cover $\mathrm{U}$, then $\mathrm{Q} \operatorname{Coh}(\mathcal{A})$ is an Abelian $k$-category and a full subcategory of $\operatorname{PreSh}(\mathrm{c}(X), \mathcal{A})$. We show that if $\mathrm{U}$ is a good $\mathcal{A}$-affine open cover of $X$, then the natural forgetful functor $\mathrm{QCoh}(\mathcal{A}) \rightarrow \operatorname{PreSh}(\mathrm{U}, \mathcal{A})$ induces an isomorphism $\operatorname{Def}_{\mathcal{F}}^{q c} \rightarrow \operatorname{Def}_{\mathcal{F}}^{U}$ of noncommutative deformation functors for any finite family $\mathcal{F}$ in $\mathrm{Q} \operatorname{Coh}(\mathcal{A})$.

Theorem 2. Let $(X, \mathcal{A})$ be a ringed space over $k$, let $\mathrm{U}$ be a good $\mathcal{A}$-affine open cover of $X$, and let $\mathcal{F}=\left\{\mathcal{F}_{1}, \ldots, \mathcal{F}_{p}\right\}$ be a finite family of quasi-coherent left $\mathcal{A}$ modules on $X$. If $\operatorname{dim}_{k} \mathrm{HH}^{n}\left(\mathrm{U}, \mathcal{A}, \mathcal{H}\right.$ om $\left.k\left(\mathcal{F}_{j}, \mathcal{F}_{i}\right)\right)<\infty$ for $1 \leq i, j \leq p, n=1,2$, then the noncommutative deformation functor $\operatorname{Def}_{\mathcal{F}}^{q c}: a_{p} \rightarrow$ Sets of $\mathcal{F}$ in $\mathrm{QCoh}(\mathcal{A})$ has a pro-representing hull $\mathrm{H}\left(\operatorname{Def}_{\mathcal{F}}^{q c}\right)$, completely determined by the $k$-linear spaces $\mathrm{HH}^{n}\left(\mathrm{U}, \mathcal{A}, \mathcal{H o m}_{k}\left(\mathcal{F}_{j}, \mathcal{F}_{i}\right)\right)$ for $1 \leq i, j \leq p, n=1,2$, together with some generalized Massey products on them.

We give examples of ringed spaces $(X, \mathcal{A})$ that admit good $\mathcal{A}$-affine open covers in section 8 . The main commutative examples are schemes $\left(X, \mathcal{O}_{X}\right)$ over $k$. The main noncommutative examples are D-schemes $(X, \mathcal{D})$ over $k$ in the sense of Beilinson, Bernstein [1]. In particular, important examples of D-schemes over an algebraically closed field $k$ of characteristic 0 include $\left(X, \mathcal{D}_{X}\right)$, where $X$ is a locally Noetherian scheme over $k$ and $\mathcal{D}_{X}$ is the sheaf of $k$-linear differential operators on $X$, and $(X, \mathrm{U}(\mathbf{g}))$, where $X$ is a separated scheme of finite type over $k$ and $\mathrm{U}(\mathrm{g})$ is the universal enveloping D-algebra of a Lie algebroid $\mathbf{g}$ on $X / k$.

Let us consider one of the Abelian categories $\mathrm{A}=\operatorname{PreSh}(\mathrm{c}, \mathcal{A}), \mathrm{A}=\operatorname{Sh}(X, \mathcal{A})$, and if $X$ has a good $\mathcal{A}$-affine open cover, $\mathrm{A}=\mathrm{Q} \operatorname{Coh}(\mathcal{A})$. We expect that the noncommutative deformation functor $\operatorname{Def}_{\mathcal{F}}^{\mathrm{A}}: \mathrm{a}_{p} \rightarrow$ Sets of $\mathcal{F}$ in $\mathrm{A}$ is controlled by $\left(\operatorname{Ext}_{\mathrm{A}}^{n}\left(\mathcal{F}_{j}, \mathcal{F}_{i}\right)\right)$ in all these cases. In fact, we show that $t\left(\operatorname{Def}_{\mathcal{F}}^{\mathrm{A}}\right)_{i j} \cong \operatorname{Ext}_{\mathrm{A}}^{1}\left(\mathcal{F}_{j}, \mathcal{F}_{i}\right)$, at least if $\operatorname{dim}_{k} \mathrm{HH}^{n}\left(\mathrm{U}, \mathcal{A}, \mathcal{H o m}_{k}\left(\mathcal{F}_{j}, \mathcal{F}_{i}\right)\right)<\infty$ for $1 \leq i, j \leq p, n=1,2$, see corollary 12. the remark in section 6, and the remark following theorem 15, It might be 
possible to develop an obstruction theory for $\operatorname{Def}_{\mathcal{F}}^{\mathrm{A}}$ with cohomology $\left(\operatorname{Ext}_{\mathrm{A}}^{n}\left(\mathcal{F}_{j}, \mathcal{F}_{i}\right)\right)$ in all these cases.

However, notice that when $\mathrm{A}=\operatorname{Sh}(X, \mathcal{A})$ or $\mathrm{A}=\mathrm{Q} \operatorname{Coh}(\mathcal{A})$ for a noncommutative sheaf of algebras $\mathcal{A}$, it is often very hard to compute $\operatorname{Ext}_{\mathrm{A}}^{n}\left(\mathcal{F}_{j}, \mathcal{F}_{i}\right)$. For instance, localization of injectives can behave badly, even when $(X, \mathcal{A})$ is a D-scheme. On the other hand, the global Hochschild cohomology groups $\mathrm{HH}^{n}\left(\mathrm{U}, \mathcal{A}, \mathcal{H} \operatorname{Hom}_{k}\left(\mathcal{F}_{j}, \mathcal{F}_{i}\right)\right)$ can be computed in concrete terms in many cases of interest.

In section 9, we give an example of this. Let $X$ be any elliptic curve over an algebraically closed field $k$ of characteristic 0 , and consider $\mathcal{O}_{X}$ as a left $\mathcal{D}_{X}$-module on $X$. We show that $\mathrm{HH}^{0}\left(\mathrm{U}, \mathcal{D}_{X}, \mathcal{E} n d_{k}\left(\mathcal{O}_{X}\right)\right) \cong k, \mathrm{HH}^{1}\left(\mathrm{U}, \mathcal{D}_{X}, \mathcal{E} n d_{k}\left(\mathcal{O}_{X}\right)\right) \cong k^{2}$, and $\mathrm{HH}^{2}\left(\mathrm{U}, \mathcal{D}_{X}, \mathcal{E} n d_{k}\left(\mathcal{O}_{X}\right)\right) \cong k$ for an open affine cover $\mathrm{U}$ of $X$ closed under intersections. Using these results and the obstruction calculus, we compute the pro-representing hull $\mathrm{H}\left(\operatorname{Def}_{\mathcal{O}_{X}}\right) \cong k \ll t_{1}, t_{2} \gg /\left(t_{1} t_{2}-t_{2} t_{1}\right)$ of the noncommutative deformation functor $\operatorname{Def}_{\mathcal{O}_{X}}$ and we also compute its versal family. In this example, it seems hard to compute $\operatorname{Ext}_{\mathcal{D}_{X}}^{n}\left(\mathcal{O}_{X}, \mathcal{O}_{X}\right)$ for $n=1,2$ in other ways.

Noncommutative deformation theory has applications to representation theory. In Laudal 14, it was shown that noncommutative deformations of modules are closely related to iterated extensions in module categories, and we used this result to study finite length categories of modules in Eriksen 4. These methods work in any Abelian $k$-category with a reasonable noncommutative deformation theory.

Acknowledgments. The author wishes to thank O.A. Laudal, A. Siqveland and R. Ile for interesting discussions while preparing this paper, and the Mittag-Leffler Institute for its hospitality during the Noncommutative geometry 2003/04 program.

\section{Functors of nOnCOMmutative Artin Rings}

Let $k$ be an algebraically closed field. We shall define the category $\mathrm{a}_{p}$ of $p$-pointed noncommutative Artin rings for any integer $p \geq 1$. For expository purposes, we first define $A_{p}$, the category of $p$-pointed algebras. An object of $A_{p}$ is an associative ring $R$, together with structural ring homomorphisms $f: k^{p} \rightarrow R$ and $g: R \rightarrow k^{p}$ such that $g \circ f=\mathrm{id}$, and a morphism $u:(R, f, g) \rightarrow\left(R^{\prime}, f^{\prime}, g^{\prime}\right)$ in $\mathrm{A}_{p}$ is ring homomorphism $u: R \rightarrow R^{\prime}$ such that $u \circ f=f^{\prime}$ and $g^{\prime} \circ u=g$.

We denote by $I=I(R)$ the ideal $I=\operatorname{ker}(g)$ for any $R \in \mathrm{A}_{p}$, and call it the radical ideal of $R$. The category $\mathrm{a}_{p}$ is the full subcategory of $\mathrm{A}_{p}$ consisting of objects $R \in \mathrm{A}_{p}$ such that $R$ is Artinian and (separated) complete in the $I$-adic topology. For any integer $n \geq 1, \mathrm{a}_{p}(n)$ is the full subcategory of $\mathrm{a}_{p}$ consisting of objects $R \in \mathrm{a}_{p}$ such that $I^{n}=0$. The pro-category $\hat{a}_{p}$ is the full subcategory of $\mathrm{A}_{p}$ consisting of objects $R \in \mathrm{A}_{p}$ such that $R_{n}=R / I^{n}$ is Artinian for all $n \geq 1$ and $R$ is (separated) complete in the $I$-adic topology. It follows that $\mathrm{a}_{p} \subseteq \hat{\mathrm{a}}_{p}$.

For any $R \in \mathrm{A}_{p}, R \in \mathrm{a}_{p}$ if and only if $\operatorname{dim}_{k} R$ is finite and $I=I(R)$ is nilpotent. If this is the case, then $I$ is the Jacobson radical of $R$, and there are $p$ isomorphism classes of simple left $R$-modules, all of dimension 1 over $k$.

For any object $R \in \mathrm{A}_{p}$, we write $e_{1}, \ldots, e_{p}$ for the indecomposable idempotents in $k^{p}$ and $R_{i j}=e_{i} R e_{j}$. Note that $R$ is a matrix ring in the sense that there is a $k$-linear isomorphism $R \cong\left(R_{i j}\right)=\oplus R_{i j}$, and multiplication in $R$ corresponds to matric multiplication in $\left(R_{i j}\right)$. In what follows, we shall denote the direct sum of any family $\left\{V_{i j}: 1 \leq i, j \leq p\right\}$ of $k$-linear vector spaces by $\left(V_{i j}\right)$.

We define a functor of (p-pointed) noncommutative Artin rings to be a covariant functor $\mathrm{D}: \mathrm{a}_{p} \rightarrow$ Sets such that $\mathrm{D}\left(k^{p}\right)=\{*\}$ is reduced to one element. It follows 
that there is a distinguished element $*_{R} \in \mathrm{D}(R)$ given by $\mathrm{D}\left(k^{p} \rightarrow R\right)(*)$ for any $R \in \mathrm{a}_{p}$. We see that $*_{R} \in \mathrm{D}(R)$ is a lifting of $* \in \mathrm{D}\left(k^{p}\right)$ to $R$, and call it the trivial lifting.

There is a natural extensions of $\mathrm{D}: \mathrm{a}_{p} \rightarrow$ Sets to the pro-category $\hat{\mathrm{a}}_{p}$, which we denote by $\mathrm{D}: \hat{\mathrm{a}}_{p} \rightarrow$ Sets. For any $R \in \hat{\mathrm{a}}_{p}$, it is given by

$$
\mathrm{D}(R)=\lim _{\longleftarrow} \mathrm{D}\left(R_{n}\right) \text {. }
$$

A pro-couple for $\mathrm{D}: \mathrm{a}_{p} \rightarrow$ Sets is a pair $(H, \xi)$ with $H \in \hat{\mathrm{a}}_{p}$ and $\xi \in \mathrm{D}(H)$, and a morphism $u:(H, \xi) \rightarrow\left(H^{\prime}, \xi^{\prime}\right)$ of pro-couples is a morphism $u: H \rightarrow H^{\prime}$ in $\hat{a}_{p}$ such that $\mathrm{D}(u)(\xi)=\xi^{\prime}$. By Yoneda's lemma, $\xi \in \mathrm{D}(H)$ corresponds to a morphism $\phi: \operatorname{Mor}(H,-) \rightarrow \mathrm{D}$ of functors on $\mathrm{a}_{p}$. We say that $(H, \xi)$ pro-represents $\mathrm{D}$ if $\phi$ is an isomorphism of functors on $\mathrm{a}_{p}$, and that $(H, \xi)$ is a pro-representing hull of $\mathrm{D}$ if $\phi: \operatorname{Mor}(H,-) \rightarrow \mathrm{D}$ is a smooth morphism of functors on $\mathrm{a}_{p}$ that induces an isomorphism of functors on $\mathrm{a}_{p}(2)$ by restriction.

Lemma 1. Let $\mathrm{D}: \mathrm{a}_{p} \rightarrow$ Sets be a functor of noncommutative Artin rings. If D has a pro-representing hull, then it is unique up to a (non-canonical) isomorphism of pro-couples.

Proof. Let $(H, \xi)$ and $\left(H^{\prime}, \xi^{\prime}\right)$ be pro-representing hulls of D, and let $\phi, \phi^{\prime}$ be the corresponding morphisms of functors on $\mathrm{a}_{p}$. By the smoothness of $\phi, \phi^{\prime}$, it follows that $\phi_{H^{\prime}}$ and $\phi_{H}^{\prime}$ are surjective. Hence there are morphisms $u:(H, \xi) \rightarrow\left(H^{\prime}, \xi^{\prime}\right)$ and $v:\left(H^{\prime}, \xi^{\prime}\right) \rightarrow(H, \xi)$ of pro-couples. Restriction to a ${ }_{p}(2)$ gives morphisms $u_{2}:\left(H_{2}, \xi_{2}\right) \rightarrow\left(H_{2}^{\prime}, \xi_{2}^{\prime}\right)$ and $v_{2}:\left(H_{2}^{\prime}, \xi_{2}^{\prime}\right) \rightarrow\left(H_{2}, \xi_{2}\right)$. But both $\left(H_{2}, \xi_{2}\right)$ and $\left(H_{2}^{\prime}, \xi_{2}^{\prime}\right)$ represent the restriction of $\mathrm{D}$ to $\mathrm{a}_{p}(2)$, so $u_{2}$ and $v_{2}$ are mutual inverses. Let us write $\operatorname{gr}_{n}(R)=I(R)^{n} / I(R)^{n+1}$ for all $R \in \hat{\mathrm{a}}_{p}$ and all $n \geq 1$. By the above argument, it follows that $\operatorname{gr}_{1}(u)$ and $\operatorname{gr}_{1}(v)$ are mutual inverses. In particular, $\operatorname{gr}_{1}(u \circ v)=\operatorname{gr}_{1}(u) \circ \operatorname{gr}_{1}(v)$ is surjective. This implies that $\operatorname{gr}_{n}(u \circ v)$ is a surjective endomorphism of the finite dimensional vector $\operatorname{space}_{\mathrm{gr}_{n}}\left(H^{\prime}\right)$ for all $n \geq 1$, and hence an automorphism of $\operatorname{gr}_{n}\left(H^{\prime}\right)$ for all $n \geq 1$. So $u \circ v$ is an automorphism, and by a symmetric argument, $v \circ u$ is an automorphism as well. It follows that $u$ and $v$ are isomorphisms of pro-couples.

For $1 \leq i, j \leq p$, let $k^{p}\left[\epsilon_{i j}\right]$ be the object in $\mathrm{a}_{p}(2)$ defined by $k^{p}\left[\epsilon_{i j}\right]=k^{p}+k \cdot \epsilon_{i j}$, with $\epsilon_{i j}=e_{i} \epsilon_{i j} e_{j}$ and $\epsilon_{i j}^{2}=0$. We define the tangent space of $\mathrm{D}: \mathrm{a}_{p} \rightarrow$ Sets to be $t(\mathrm{D})=\left(t(\mathrm{D})_{i j}\right)$ with $t(\mathrm{D})_{i j}=\mathrm{D}\left(k^{p}\left[\epsilon_{i j}\right]\right)$ for $1 \leq i, j \leq p$. Note that if $(H, \xi)$ is a pro-representing hull for $\mathrm{D}$, then $\xi$ induces a bijection $t(\mathrm{D})_{i j} \cong I(H)_{i j} / I(H)_{i j}^{2}$. In particular, $t(\mathrm{D})_{i j}$ has a canonical $k$-linear structure in this case.

A small surjection in $\mathrm{a}_{p}$ is a surjective morphism $u: R \rightarrow S$ in $\mathrm{a}_{p}$ such that $K I=I K=0$, where $I=I(R)$ and $K=\operatorname{ker}(u)$. Given a functor $\mathrm{D}: \mathrm{a}_{p} \rightarrow$ Sets of noncommutative Artin rings, a small lifting situation for D is defined by a small surjection $u: R \rightarrow S$ in $\mathrm{a}_{p}$ and an element $\xi_{S} \in \mathrm{D}(S)$. In order to study the existence of, and ultimately construct, a pro-representing hull $H$ for D, we are led to consider the possible liftings of $\xi_{S}$ to $R$ in small lifting situations.

Let $\left\{\mathrm{H}_{i j}^{n}: 1 \leq i, j \leq p\right\}$ be a family of vector spaces over $k$ for $n=1,2$. We say that a functor $\mathrm{D}: \mathrm{a}_{p} \rightarrow$ Sets of noncommutative Artin rings has an obstruction theory with cohomology $\left(\mathrm{H}_{i j}^{n}\right)$ if the following conditions hold:

(1) For any small lifting situation, given by a small surjection $u: R \rightarrow S$ in a $_{p}$ with kernel $K=\operatorname{ker}(u)$ and an element $\xi_{S} \in \mathrm{D}(S)$, we have: 
(a) There exists a canonical obstruction $o\left(u, \xi_{S}\right) \in\left(\mathrm{H}_{i j}^{2} \otimes_{k} K_{i j}\right)$ such that $o\left(u, \xi_{S}\right)=0$ if and only if there exists a lifting of $\xi_{S}$ to $R$,

(b) If $o\left(u, \xi_{S}\right)=0$, there is an transitive and effective action of $\left(\mathrm{H}_{i j}^{1} \otimes_{k} K_{i j}\right)$ on the set of liftings of $\xi_{S}$ to $R$.

(2) Let $u_{i}: R_{i} \rightarrow S_{i}$ be a small surjection with kernel $K_{i}=\operatorname{ker}\left(u_{i}\right)$ and let $\xi_{i} \in \mathrm{D}\left(S_{i}\right)$ for $i=1,2$. If $\alpha: R_{1} \rightarrow R_{2}$ and $\beta: S_{1} \rightarrow S_{2}$ are morphisms in a $p$ such that $u_{2} \circ \alpha=\beta \circ u_{1}$ and $\mathrm{D}(\beta)\left(\xi_{1}\right)=\xi_{2}$, then $\alpha^{*}\left(o\left(u_{1}, \xi_{1}\right)\right)=o\left(u_{2}, \xi_{2}\right)$, where $\alpha^{*}:\left(\mathrm{H}_{i j}^{2} \otimes_{k} K_{1, i j}\right) \rightarrow\left(\mathrm{H}_{i j}^{2} \otimes_{k} K_{2, i j}\right)$ is the natural map induced by $\alpha$.

Moreover, if $\mathrm{H}_{i j}^{n}$ has finite $k$-dimension for $1 \leq i, j \leq p, n=1,2$, then we say that $\mathrm{D}$ has an obstruction theory with finite dimensional cohomology $\left(\mathrm{H}_{i j}^{n}\right)$.

In the rest of this section, we shall assume that $D: a_{p} \rightarrow$ Sets is a functor of noncommutative Artin rings that has an obstruction theory with finite dimensional cohomology $\left(\mathrm{H}_{i j}^{n}\right)$. Note that for any object $R \in \mathrm{a}_{p}(2)$, the morphism $R \rightarrow k^{p}$ is a small surjection. This implies that there is a canonical set-theoretical bijection

$$
\left(\mathrm{H}_{i j}^{1} \otimes_{k} I(R)_{i j}\right) \cong \mathrm{D}(R)
$$

given by the trivial lifting $*_{R} \in \mathrm{D}(R)$. In particular, there is a set-theoretical bijection between $H_{i j}^{1}$ and $t(\mathrm{D})_{i j}=\mathrm{D}\left(k^{p}\left[\epsilon_{i j}\right]\right)$ for $1 \leq i, j \leq p$.

We define $T^{n}$ to be the free, formal matrix ring in $\hat{\mathrm{a}}_{p}$ generated by the $k$-linear vector spaces $\left\{\left(\mathrm{H}_{i j}^{n}\right)^{*}: 1 \leq i, j \leq p\right\}$ for $n=1,2$, where $\left(\mathrm{H}_{i j}^{n}\right)^{*}=\operatorname{Hom}_{k}\left(\mathrm{H}_{i j}^{n}, k\right)$. For any $R \in \mathrm{a}_{p}(2)$, we have natural isomorphisms

$$
\mathrm{D}(R) \cong\left(\mathrm{H}_{i j}^{1} \otimes_{k} I(R)_{i j}\right) \cong\left(\operatorname{Hom}_{k}\left(\left(\mathrm{H}_{i j}^{1}\right)^{*}, I(R)_{i j}\right)\right) \cong \operatorname{Mor}\left(T_{2}^{1}, R\right),
$$

where $T_{2}^{1}=T^{1} / I\left(T^{1}\right)^{2}$. It follows that there is an isomorphism $\operatorname{Mor}\left(T_{2}^{1},-\right) \rightarrow \mathrm{D}$ of functors on $\mathrm{a}_{p}(2)$, i.e. the restriction of $\mathrm{D}$ to $\mathrm{a}_{p}(2)$ is represented by $\left(T_{2}^{1}, \xi_{2}\right)$ for some $\xi_{2} \in \mathrm{D}\left(T_{2}^{1}\right)$.

Theorem 2. Let $\mathrm{D}: \mathrm{a}_{p} \rightarrow$ Sets be a functor of noncommutative Artin rings. If $\mathrm{D}$ has an obstruction theory with finite dimensional cohomology, then there is an obstruction morphism o: $T^{2} \rightarrow T^{1}$ in $\hat{\mathrm{a}}_{p}$ such that $H=T^{1} \widehat{\otimes}_{T^{2}} k^{p}$ is a prorepresenting hull of $\mathrm{D}$.

Proof. Let us write $I=I\left(T^{1}\right), T_{n}^{1}=T^{1} / I^{n}$ and $t_{n}: T_{n+1}^{1} \rightarrow T_{n}^{1}$ for the natural morphism for all $n \geq 1$. Let $a_{2}=I^{2}$ and $H_{2}=T^{1} / a_{2}=T_{2}^{1}$, then the restriction of $\mathrm{D}$ to $\mathrm{a}_{p}(2)$ is represented by $\left(H_{2}, \xi_{2}\right)$ and $H_{2} \cong T_{2}^{1} \otimes_{T^{2}} k^{p}$. Using $o_{2}$ and $\xi_{2}$ as a starting point, we shall construct $o_{n+1}$ and $\xi_{n+1}$ for $n \geq 2$ inductively. So let $n \geq 2$, and assume that the morphism $o_{n}: T^{2} \rightarrow T_{n}^{1}$ and the deformation $\xi_{n} \in \mathrm{D}\left(H_{n}\right)$ are given, with $H_{n}=T_{n}^{1} \otimes_{T^{2}} k^{p}$. We may assume that $t_{n-1} \circ o_{n}=o_{n-1}$ and that $\xi_{n}$ is a lifting of $\xi_{n-1}$.

Let us first construct the morphism $o_{n+1}: T^{2} \rightarrow T_{n+1}^{1}$. We define $a_{n}^{\prime}$ to be the ideal in $T_{n}^{1}$ generated by $o_{n}\left(I\left(T^{2}\right)\right)$. Then $a_{n}^{\prime}=a_{n} / I^{n}$ for an ideal $a_{n} \subseteq T^{1}$ with $I^{n} \subseteq a_{n}$, and $H_{n} \cong T^{1} / a_{n}$. Let $b_{n}=I a_{n}+a_{n} I$, then we obtain the following commutative diagram:

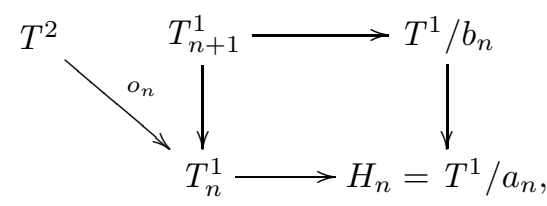


There is an obstruction $o_{n+1}^{\prime}=o\left(T^{1} / b_{n} \rightarrow H_{n}, \xi_{n}\right)$ for lifting $\xi_{n}$ to $T^{1} / b_{n}$ since $T^{1} / b_{n} \rightarrow T^{1} / a_{n}$ is a small surjection, hence a morphism $o_{n+1}^{\prime}: T^{2} \rightarrow T^{1} / b_{n}$. Let $a_{n+1}^{\prime \prime}$ be the ideal in $T^{1} / b_{n}$ generated by $o_{n+1}^{\prime}\left(I\left(T^{2}\right)\right)$. Then $a_{n+1}^{\prime \prime}=a_{n+1} / b_{n}$ for an ideal $a_{n+1} \subseteq T^{1}$ with $b_{n} \subseteq a_{n+1} \subseteq a_{n}$. Let $H_{n+1}=T^{1} / a_{n+1}$, then we obtain the following commutative diagram:

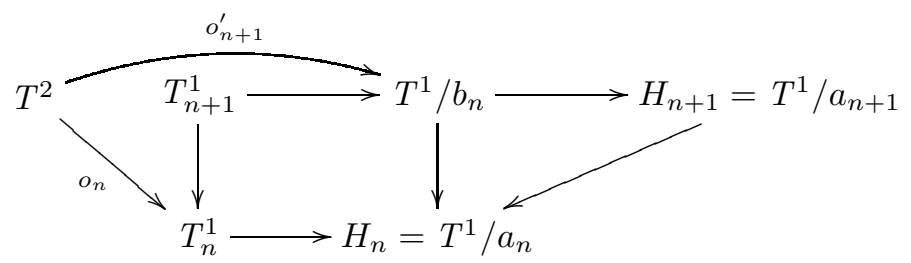

By the choice of $a_{n+1}$, the obstruction for lifting $\xi_{n}$ to $H_{n+1}$ is zero. We can therefore find a lifting $\xi_{n+1} \in \mathrm{D}\left(H_{n+1}\right)$ of $\xi_{n}$ to $H_{n+1}$.

We claim that there is a morphism $o_{n+1}: T^{2} \rightarrow T_{n+1}^{1}$ that commutes with $o_{n+1}^{\prime}$ and $o_{n}$. Note that $a_{n-1}=I^{n-1}+a_{n}$ since $t_{n-1} \circ o_{n}=o_{n-1}$. For simplicity, we write $O(K)=\left(\operatorname{Hom}_{k}\left(\operatorname{gr}_{1}\left(T^{2}\right)_{i j}, K_{i j}\right)\right)$ for any family $K=\left(K_{i j}\right)$ of vector spaces over $k$. The following diagram of $k$-vector spaces is commutative with exact columns:

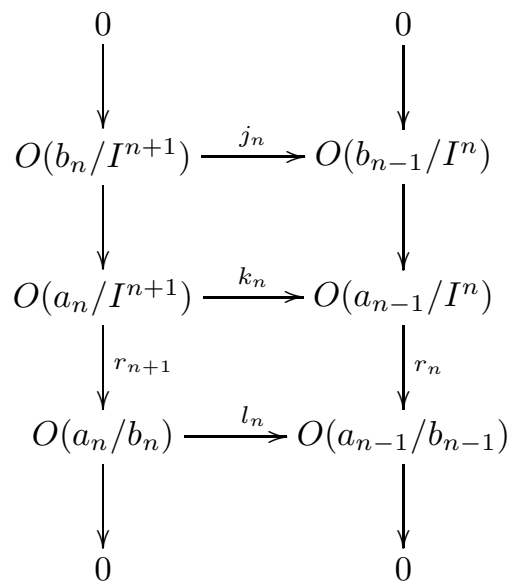

We may consider $o_{n}$ as an element in $O\left(a_{n-1} / I^{n}\right)$, and $o_{n+1}^{\prime} \in O\left(a_{n} / b_{n}\right)$. Since $o_{n}^{\prime}$ commutes with $o_{n+1}^{\prime}$ and $o_{n}$, we get $l_{n}\left(o_{n+1}^{\prime}\right)=r_{n}\left(o_{n}\right)$. To prove the claim, it is enough to find an element $o_{n+1} \in O\left(a_{n} / I^{n+1}\right)$ such that $r_{n+1}\left(o_{n+1}\right)=o_{n+1}^{\prime}$ and $k_{n}\left(o_{n+1}\right)=o_{n}$. Since $o_{n}\left(I\left(T^{2}\right)\right) \subseteq a_{n}$, there is an element $\bar{o}_{n+1} \in O\left(a_{n} / I^{n+1}\right)$ such that $k_{n}\left(\bar{o}_{n+1}\right)=o_{n}$. But $a_{n-1}=a_{n}+I^{n-1}$ implies that $j_{n}$ is surjective, so the claim follows from the snake lemma. In particular, $T_{n+1}^{1} \otimes_{T^{2}} k^{p} \cong H_{n+1}$ when the tensor product is taken over $o_{n+1}$.

By induction, we find a morphism $o_{n}: T^{2} \rightarrow T_{n}^{1}$ and an element $\xi_{n} \in \mathrm{D}\left(H_{n}\right)$ for all integers $n \geq 1$, with $H_{n}=T_{n}^{1} \otimes_{T^{2}} k^{p}$. Using the universal property of the projective limit, we obtain a morphism $o: T^{2} \rightarrow T^{1}$ in $\hat{\mathrm{a}}_{p}$ and an element $\xi \in \mathrm{D}(H)$, with $H=T^{1} \hat{\otimes}_{T^{2}} k^{p}$. We claim that $(H, \xi)$ is a pro-representable hull for $\mathrm{D}$.

Clearly, it is enough to prove that $\left(H_{n}, \xi_{n}\right)$ is a pro-representing hull for the restriction of $\mathrm{D}$ to $\mathrm{a}_{p}(n)$ for all $n \geq 3$. So let $\phi_{n}: \operatorname{Mor}\left(H_{n},-\right) \rightarrow \mathrm{D}$ be the morphism of functors on $\mathrm{a}_{p}(n)$ corresponding to $\xi_{n}$ for some $n \geq 3$. We shall 
prove that $\phi_{n}$ is a smooth morphism. Let $u: R \rightarrow S$ be a small surjection in $\mathrm{a}_{p}(n)$ with kernel $K$, let $E_{R} \in \mathrm{D}(R)$ and $v \in \operatorname{Mor}\left(H_{n}, S\right)$ be elements such that $\mathrm{D}(u)\left(E_{R}\right)=\mathrm{D}(v)\left(\xi_{n}\right)=E_{S}$, and consider the following commutative diagram:

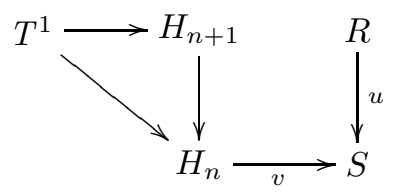

We can find a morphism $v^{\prime}: T^{1} \rightarrow R$ that makes the diagram commutative. This implies that $v^{\prime}\left(a_{n}\right) \subseteq K$, and since $u$ is small, that $v^{\prime}\left(b_{n}\right)=0$. But the induced map $T^{1} / b_{n} \rightarrow R$ maps the obstruction $o_{n+1}^{\prime}$ to $o\left(u, E_{S}\right)=0$. It follows that $v^{\prime}\left(a_{n+1}\right)=0$, hence $v^{\prime}$ induces a morphism $v^{\prime}: H_{n+1} \rightarrow R$ making the diagram commutative. Since $v^{\prime}\left(I\left(H_{n+1}\right)^{n}\right)=0$, we may consider $v^{\prime}$ as a map from $H_{n+1} / I\left(H_{n+1}\right)^{n} \cong H_{n}$. This proves that there is a morphism $v^{\prime}: H_{n} \rightarrow R$ such that $u \circ v^{\prime}=v$.

Let $E_{R}^{\prime}=\mathrm{D}\left(v^{\prime}\right)\left(\xi_{n}\right)$, then $E_{R}^{\prime}$ is a lifting of $E_{S}$ to $R$, and the difference between $E_{R}$ and $E_{R}^{\prime}$ is given by an element $d \in\left(\mathrm{H}_{i j}^{1} \otimes_{k} K_{i j}\right)=\left(\operatorname{Hom}_{k}\left(\operatorname{gr}_{1}\left(T^{1}\right)_{i j}, K_{i j}\right)\right)$. Let $v^{\prime \prime}: T^{1} \rightarrow R$ be the morphism given by $v^{\prime \prime}\left(x_{i j}(l)\right)=v^{\prime}\left(x_{i j}(l)\right)+d\left(\overline{x_{i j}(l)}\right)$, where $\left\{x_{i j}(l): 1 \leq l \leq d_{i j}\right\}$ is a basis for $\mathrm{H}_{i j}^{1}$ for $1 \leq i, j \leq p$. Since $a_{n+1} \subseteq I^{2}$ and $u$ is small, $v^{\prime \prime}\left(a_{n+1}\right) \subseteq v^{\prime}\left(a_{n+1}\right)+I(R) K+K I(R)+K^{2}=v^{\prime}\left(a_{n+1}\right)=0$. This implies that $v^{\prime \prime}$ induces a morphism $v^{\prime \prime}: H_{n} \rightarrow R$. By construction, $u \circ v^{\prime \prime}=u \circ v^{\prime}=v$ and $\mathrm{D}\left(v^{\prime \prime}\right)\left(\xi_{n}\right)=E_{R}$, and this proves that $\phi_{n}$ is smooth.

We remark that a more general version of theorem 2 can be proved if $\mathrm{D}$ has an obstruction theory with cohomology $\left(\mathrm{H}_{i j}^{n}\right)$ and $\mathrm{H}_{i j}^{1}$ has a countable $k$-basis for $1 \leq i, j \leq p$, using the methods of Laudal [15].

Corollary 3. Let $\mathrm{D}: \mathrm{a}_{p} \rightarrow$ Sets be a functor of noncommutative Artin rings. If $\mathrm{D}$ has an obstruction theory with finite dimensional cohomology $\left(\mathrm{H}_{i j}^{n}\right)$, then there is a $k$-linear isomorphism $\mathrm{H}_{i j}^{1} \cong t(\mathrm{D})_{i j}$ for $1 \leq i, j \leq p$.

Corollary 4. Let D : $\mathrm{a}_{p} \rightarrow$ Sets be a functor of noncommutative Artin rings. If $\mathrm{D}$ has an obstruction theory with finite dimensional cohomology $\left(\mathrm{H}_{i j}^{n}\right)$, then the pro-representing hull $\mathrm{H}(\mathrm{D})$ is completely determined by the k-linear spaces $\mathrm{H}_{i j}^{n}$ for $1 \leq i, j \leq p, n=1,2$, together with some generalized Massey products on them.

These results are natural generalizations of similar results for functors of commutative Artin rings. As in the commutative case, the generalized Massey product structure on $\mathrm{H}_{i j}^{n}$ can be considered as the $k$-linear dual of the obstruction morphism $o: T^{2} \rightarrow T^{1}$, see Laudal [15, [13. If $\mathrm{D}$ is obstructed, i.e. $o\left(I\left(T^{2}\right)\right) \neq 0$, then it is a non-trivial task to compute $\mathrm{H}(\mathrm{D})$ using generalized Massey products.

Let $\mathrm{D}: \mathrm{a}_{p} \rightarrow$ Sets be a functor of noncommutative Artin rings. For $1 \leq i \leq p$, we write $\mathrm{D}_{i}: \mathrm{a}_{1} \rightarrow$ Sets for the restriction of $\mathrm{D}$ to $\mathrm{a}_{1}$ using the $i$ 'th natural inclusion of categories $\mathrm{a}_{1} \hookrightarrow \mathrm{a}_{p}$, and $\mathrm{D}_{i}^{c}: \mathrm{I} \rightarrow$ Sets for the restriction of $\mathrm{D}_{i}$ to I, where I is the full subcategory of $\mathrm{a}_{1}$ consisting of commutative algebras. For any associative ring $R$, we define the commutativization of $R$ to be the quotient ring $R^{c}=R / I^{c}(R)$, where $I^{c}(R)$ is the ideal in $R$ generated by the set of commutators $\{a b-b a: a, b \in R\}$.

Proposition 5. Let $\mathrm{D}: \mathrm{a}_{p} \rightarrow$ Sets be a functor of noncommutative Artin rings. If $\mathrm{D}$ has an obstruction theory with finite dimensional cohomology $\left(\mathrm{H}_{i j}^{n}\right)$, then $\mathrm{D}_{i}^{c}$ has a commutative hull $\mathrm{H}\left(\mathrm{D}_{i}^{c}\right) \cong \mathrm{H}\left(\mathrm{D}_{i}\right)^{c}$ for $1 \leq i \leq p$, and $\mathrm{H}(\mathrm{D})^{c} \cong \oplus \mathrm{H}\left(\mathrm{D}_{i}^{c}\right)$. 
Proof. Clearly, the functor $\mathrm{D}_{i}$ has an obstruction theory with finite dimensional cohomology $\mathrm{H}_{i i}^{n}$ for $1 \leq i \leq p$, and therefore a pro-representing hull $\mathrm{H}\left(\mathrm{D}_{i}\right)$ that is determined by an obstruction morphism $o_{i}: T^{2} \rightarrow T^{1}$. Similarly, the functor $\mathrm{D}_{i}^{c}$ has an obstruction theory with finite dimensional cohomology $\mathrm{H}_{i i}^{n}$ for $1 \leq i \leq p$, and therefore a pro-representing hull $\mathrm{H}\left(\mathrm{D}_{i}^{c}\right)$ that is determined by an obstruction morphism $o_{i}^{c}:\left(T^{2}\right)^{c} \rightarrow\left(T^{1}\right)^{c}$. These morphisms are defined by obstructions in small lifting situations, so it follows from the functorial nature of the obstructions that $o_{i}$ and $o_{i}^{c}$ are compatible. Hence $\mathrm{H}\left(\mathrm{D}_{i}^{c}\right) \cong \mathrm{H}\left(\mathrm{D}_{i}\right)^{c}$ for $1 \leq i \leq p$. For the second part, note that $\mathrm{H}(\mathrm{D})_{i j}^{c}=0$ whenever $i \neq j$. In fact, for any $x_{i j} \in \mathrm{H}(\mathrm{D})_{i j}$ with $i \neq j$, the commutator $\left[e_{i}, x_{i j}\right]=e_{i} x_{i j}-x_{i j} e_{i}=x_{i j}$ is zero in $\mathrm{H}(\mathrm{D})^{c}$. This implies that $\mathrm{H}(\mathrm{D})^{c}=\oplus \mathrm{H}(\mathrm{D})_{i i}^{c} \cong \oplus \mathrm{H}\left(\mathrm{D}_{i}\right)^{c} \cong \oplus \mathrm{H}\left(\mathrm{D}_{i}^{c}\right)$.

\section{Noncommutative Deformation FUnCtors}

Let $k$ be an algebraically closed field, and let A be any Abelian $k$-category. For any object $X \in \mathrm{A}$, we recall the definition of $\operatorname{Def}_{X}^{\mathrm{A}}: \mathrm{I} \rightarrow$ Sets, the commutative deformation functor of $X$ in $\mathrm{A}$, which is a functor of Artin rings, and discuss how to generalize this definition to noncommutative deformation functors $\operatorname{Def}_{X}^{A}: a_{p} \rightarrow$ Sets of a family $X=\left\{X_{1}, \ldots, X_{p}\right\}$ in the category A.

Let $R$ be any object in $\mathrm{a}_{p}$. We consider the category $\mathrm{A}_{R}$ of $R$-objects in $\mathrm{A}$, i.e. the category with objects $(X, \phi)$, where $X$ is an object of $\mathrm{A}$ and $\phi: R \rightarrow \operatorname{Mor}_{\mathrm{A}}(X, X)$ is a $k$-algebra homomorphism, and with morphisms $f:(X, \phi) \rightarrow\left(X^{\prime}, \phi^{\prime}\right)$, where $f: X \rightarrow X^{\prime}$ is a morphism in A such that $f \circ \phi(r)=\phi^{\prime}(r) \circ f$ for all $r \in R$. Clearly, $\mathrm{A}_{R}$ is an Abelian $R$-category.

Let $R$ be any object of $\mathrm{a}_{p}$, let $\mathrm{B}$ be any Abelian $R$-category, and let $\bmod (R)$ be the category of finitely generated left $R$-modules. For each object $Y \in \mathrm{B}$, there is a unique finite colimit preserving functor $Y \otimes_{R}-: \bmod (R) \rightarrow \mathrm{B}$ that maps $R$ to $Y$, given in the following way: If $M=\operatorname{coker}(f)$, where $f: R^{m} \rightarrow R^{n}$ is a homomorphism of left $R$-modules, then $Y \otimes_{R} M=\operatorname{coker}(F)$, where $F: Y^{m} \rightarrow Y^{n}$ is the morphism in B induced by $f$ and the $R$-linear structure on B. We say that an object $Y \in \mathrm{B}$ is $R$-flat if $Y \otimes_{R}$ - is exact. It is clear that any morphism $u: R \rightarrow S$ in $\mathrm{a}_{p}$ induces a functor $-\otimes_{R} S: \mathrm{A}_{R} \rightarrow \mathrm{A}_{S}$.

Given an object $X \in \mathrm{A}$, the flat deformation functor $\operatorname{Def}_{X^{\prime}}^{\mathrm{A}}: I \rightarrow$ Sets is given in the following way: For any object $R \in \mathrm{I}$, we define a lifting of $X$ to $R$ to be an object $X_{R} \in \mathrm{A}_{R}$ which is $R$-flat, together with an isomorphism $\eta: X_{R} \otimes_{R} k \rightarrow X$ in $\mathrm{A}$, and we say that two liftings $\left(X_{R}, \eta\right)$ and $\left(X_{R}^{\prime}, \eta^{\prime}\right)$ are equivalent if there is an isomorphism $\tau: X_{R} \rightarrow X_{R}^{\prime}$ in $\mathrm{A}_{R}$ such that $\eta^{\prime} \circ\left(\tau \otimes_{R}\right.$ id $)=\eta$. Let $\operatorname{Def}_{X}^{\mathrm{A}}(R)$ be the set of equivalence classes of liftings of $X$ to $R$. Then $\operatorname{Def}_{X}^{A}: I \rightarrow$ Sets is a functor of Artin rings, called the commutative deformation functor of $X$ in A.

When $\mathrm{A}=\operatorname{Mod}(A)$, the category of left modules over an associative $k$-algebra $A$, we remark that the category $\mathrm{A}_{R}$ is the category of $A-R$ bimodules on which $k$ acts centrally, and the tensor product defined above is the usual tensor product over $R$. It follows that the usual deformation functor $\operatorname{Def}_{M}$ of a left $A$-module $M$ coincides with the deformation functor defined above.

Given a finite family $X=\left\{X_{1}, \ldots, X_{p}\right\}$ of objects in A, we would like to define a noncommutative deformation functor $\operatorname{Def}_{X}^{\mathrm{A}}: \mathrm{a}_{p} \rightarrow$ Sets of the family $X$ in A. When $\mathrm{A}=\operatorname{Mod}(A)$, the category of left modules over an associative $k$-algebra $A$, such a deformation functor was defined in Laudal [14. The idea is to replace the 
condition that $M_{R}$ is a flat right $R$-module with the matric freeness condition that

$$
M_{R} \cong\left(M_{i} \otimes_{k} R_{i j}\right)
$$

as right $R$-modules. This is reasonable, since an $R$-module is flat if and only if (1) holds when $R \in \mathrm{I}$ or $R \in \mathrm{a}_{1}$, see Bourbaki [3], Corollary II.3.2. However, it is not clear whether an $R$-module is flat if and only if (11) holds when $R \in \mathrm{a}_{p}$ for $p \geq 2$.

We choose to define noncommutative deformation functors using Laudal's matric freeness condition rather than flatness. However, it is not completely clear how to do this for an arbitrary Abelian $k$-category. We shall therefore restrict our attention to categories of sheaves and presheaves of modules.

Let c be a small category, let $\mathcal{A}$ be a presheaf of $k$-algebras on c, and assume that $\mathrm{A}$ is an Abelian $k$-category and a full subcategory of the category $\operatorname{PreSh}(\mathrm{c}, \mathcal{A})$ of presheaves of left $\mathcal{A}$-modules on c. Then there is a forgetful functor $\pi_{c}: \mathrm{A} \rightarrow \operatorname{Mod}(k)$ for each object $c \in \mathrm{c}$ and an induced forgetful functor $\pi_{c}^{R}: \mathrm{A}_{R} \rightarrow \operatorname{Mod}(R)$ for each object $c \in \mathrm{c}$ and each $R \in \mathrm{a}_{p}$. We say that an object $X_{R} \in \mathrm{A}_{R}$ is $R$-free if $\pi_{c}^{R}\left(X_{R}\right) \cong\left(\pi_{c}\left(X_{i}\right) \otimes_{k} R_{i j}\right)$ in $\operatorname{Mod}(R)$ for all objects $c \in \mathrm{c}$.

Let $\mathrm{A}$ be an Abelian $k$-category, and let $X=\left\{X_{1}, \ldots, X_{p}\right\}$ be a finite family of objects in $\mathrm{A}$. If $\mathrm{A}$ is a full subcategory of $\operatorname{PreSh}(c, \mathcal{A})$, we define the noncommutative deformation functor $\operatorname{Def}_{X}^{\mathrm{A}}: \mathrm{a}_{p} \rightarrow$ Sets in the following way: A lifting of $X$ to $R$ is an object $X_{R}$ in $\mathrm{A}_{R}$ that is R-free, together with isomorphisms $\eta_{i}: X_{R} \otimes_{R} k_{i} \rightarrow X_{i}$ in $\mathrm{A}$ for $1 \leq i \leq p$, and two liftings $\left(X_{R}, \eta_{i}\right)$ and $\left(X_{R}^{\prime}, \eta_{i}^{\prime}\right)$ are equivalent if there is an isomorphism $\tau: X_{R} \rightarrow X_{R}^{\prime}$ in $\mathrm{A}_{R}$ such that $\eta_{i}^{\prime} \circ\left(\tau \otimes_{R}\right.$ id $)=\eta_{i}$ for $1 \leq i \leq p$. Let $\operatorname{Def}_{X}^{\mathrm{A}}(R)$ be the set of equivalence classes of liftings of $X$ to $R$. Then $\operatorname{Def}_{X}^{\mathrm{A}}$ is a functor of noncommutative Artin rings, the noncommutative deformation functor of the family $X$ in A. When the category $\mathrm{A}$ is understood from the context, we often write $\operatorname{Def}_{X}$ for $\operatorname{Def}_{X}^{\mathrm{A}}$.

\section{Deformations of Presheaves of modules}

Let $k$ be an algebraically closed field. The category $\operatorname{PreSh}(\mathrm{c}, \mathcal{A})$ of presheaves of left $\mathcal{A}$-modules on $\mathrm{c}$ is an Abelian $k$-category for any small category $\mathrm{c}$ and any presheaf $\mathcal{A}$ of associative $k$-algebras on c, and we shall consider deformations in this category. To fix notations, a presheaf on $\mathrm{c}$ is always covariant in this paper.

For any finite family $\mathcal{F}=\left\{\mathcal{F}_{1}, \ldots, \mathcal{F}_{p}\right\}$ of presheaves of left $\mathcal{A}$-modules on c, we consider the noncommutative deformation functor $\operatorname{Def}_{\mathcal{F}}: \mathrm{a}_{p} \rightarrow$ Sets, defined by $\operatorname{Def}_{\mathcal{F}}=\operatorname{Def}_{\mathcal{F}}^{\mathrm{A}}$ with $\mathrm{A}=\operatorname{PreSh}(\mathrm{c}, \mathcal{A})$. We shall describe this functor in concrete terms.

Let $R \in \mathrm{a}_{p}$, and consider a lifting $\mathcal{F}_{R}$ of the family $\mathcal{F}$ to $R$. Without loss of generality, we may assume that $\mathcal{F}_{R}(c)=\left(\mathcal{F}_{i}(c) \otimes_{k} R_{i j}\right)$ with the natural right $R$ module structure for all $c \in \mathrm{c}$. To describe the lifting completely, we must specify the left action of $\mathcal{A}(c)$ on $\left(\mathcal{F}_{i}(c) \otimes_{k} R_{i j}\right)$ for any object $c \in \mathrm{c}$, and the restriction map $\mathcal{F}_{R}(\phi):\left(\mathcal{F}_{i}(c) \otimes_{k} R_{i j}\right) \rightarrow\left(\mathcal{F}_{i}\left(c^{\prime}\right) \otimes_{k} R_{i j}\right)$ for any morphism $\phi: c \rightarrow c^{\prime}$ in c. It is enough to specify the action of $a \in \mathcal{A}(c)$ on elements of the form $f_{j} \otimes e_{j}$ in $\mathcal{F}_{j}(c) \otimes_{k} R_{j j}$, and we must have

$$
a\left(f_{j} \otimes e_{j}\right)=\left(a f_{j}\right) \otimes e_{j}+\sum f_{i}^{\prime} \otimes r_{i j}
$$

with $f_{i}^{\prime} \in \mathcal{F}_{i}(c), r_{i j} \in I(R)_{i j}$ for all objects $c \in \mathrm{c}$. Similarly, it is enough to specify the restriction map $\mathcal{F}_{R}(\phi)$ on elements of the form $f_{j} \otimes e_{j}$ in $\mathcal{F}_{j}(c) \otimes_{k} R_{j j}$, and we 
must have

$$
\mathcal{F}_{R}(\phi)\left(f_{j} \otimes e_{j}\right)=\mathcal{F}_{j}(\phi)\left(f_{j}\right) \otimes e_{j}+\sum f_{i}^{\prime} \otimes r_{i j}
$$

with $f_{i}^{\prime} \in \mathcal{F}_{i}\left(c^{\prime}\right), r_{i j} \in I(R)_{i j}$ for all morphisms $\phi: c \rightarrow c^{\prime}$ in c.

Let $Q^{R}\left(c, c^{\prime}\right)=\left(\operatorname{Hom}_{k}\left(\mathcal{F}_{j}(c), \mathcal{F}_{i}\left(c^{\prime}\right) \otimes_{k} R_{i j}\right)\right)$ for all objects $c, c^{\prime} \in \mathrm{c}$, and write $Q^{R}(c)=Q^{R}(c, c)$. There is a natural product $Q^{R}\left(c^{\prime}, c^{\prime \prime}\right) \otimes_{k} Q^{R}\left(c, c^{\prime}\right) \rightarrow Q^{R}\left(c, c^{\prime \prime}\right)$ for all objects $c, c^{\prime}, c^{\prime \prime} \in \mathrm{c}$, given by composition of maps and multiplication in $R$, such that $Q^{R}(c)$ is an associative $k$-algebra and $Q^{R}\left(c, c^{\prime}\right)$ is an $Q^{R}\left(c^{\prime}\right)-Q^{R}(c)$ bimodule in a natural way.

Lemma 6. For any $R \in \mathrm{a}_{p}$, there is a bijective correspondence between the following data, up to equivalence, and $\operatorname{Def}_{\mathcal{F}}(R)$ :

(1) For any $c \in \mathrm{c}$, a k-algebra homomorphism $L(c): \mathcal{A}(c) \rightarrow Q^{R}(c)$ that satisfies equation (2),

(2) For any morphism $\phi: c \rightarrow c^{\prime}$ in $c$, an element $L(\phi) \in Q^{R}\left(c, c^{\prime}\right)$ that satisfies equation (3) and $L(\phi) L(c)=L\left(c^{\prime}\right) L(\phi)$,

(3) We have $L(\mathrm{id})=\mathrm{id}$ and $L\left(\phi^{\prime}\right) L(\phi)=L\left(\phi^{\prime} \circ \phi\right)$ for all morphisms $\phi: c \rightarrow c^{\prime}$ and $\phi^{\prime}: c^{\prime} \rightarrow c^{\prime \prime}$ in $\mathrm{c}$.

For any $R \in \mathrm{a}_{p}$, we may for any $c \in \mathrm{c}$ consider $L(c): \mathcal{A}(c) \rightarrow Q^{R}(c)$ given by $L(c)(a)\left(f_{j}\right)=a f_{j} \otimes e_{j}$ for all $a \in \mathcal{A}(c), f_{j} \in \mathcal{F}_{j}(c)$, and for any morphism $\phi: c \rightarrow c^{\prime}$ in c consider $L(\phi) \in Q^{R}\left(c, c^{\prime}\right)$ given by $L(\phi)\left(f_{j}\right)=\mathcal{F}_{j}(\phi)\left(f_{j}\right) \otimes e_{j}$ for all $f_{j} \in \mathcal{F}_{j}(c)$. These data correspond to the trivial deformation $*_{R} \in \operatorname{Def}_{\mathcal{F}}(R)$.

Lemma 7. There is a bijection $\phi_{i j}: t\left(\operatorname{Def}_{\mathcal{F}}\right)_{i j} \rightarrow \operatorname{Ext}_{\mathcal{A}}^{1}\left(\mathcal{F}_{j}, \mathcal{F}_{i}\right)$ for $1 \leq i, j \leq p$ that maps trivial deformations to split extensions.

Proof. Let $R=k^{p}\left[\epsilon_{i j}\right]$, and let $\mathcal{F}_{R}$ be a lifting of $\mathcal{F}$ to $R$. We consider the $j$ 'th column $\mathcal{F}_{R}^{j}$ of $\mathcal{F}_{R}$, given by $c \mapsto \mathcal{F}_{R}(c) e_{j}$, which is a sub-presheaf of $\mathcal{F}_{R}$ of left $\mathcal{A}$-modules on c since $\mathcal{F}_{R}^{j}(c)$ is invariant under $L(c)$ and $L(\phi)$ for any $c \in \mathrm{c}$ and any $\phi: c \rightarrow c^{\prime}$ in c. Moreover, there is a natural exact sequence $0 \rightarrow \mathcal{F}_{i} \rightarrow \mathcal{F}_{R}^{j} \rightarrow \mathcal{F}_{j} \rightarrow 0$ in $\operatorname{PreSh}(\mathrm{c}, \mathcal{A})$, since $\mathcal{F}_{R}^{j}(c)=\mathcal{F}_{j}(c) \oplus \mathcal{F}_{i}(c) \epsilon_{i j}$ for all $c \in$ c. Clearly, equivalent liftings of $\mathcal{F}$ to $R$ give equivalent extensions in $\operatorname{PreSh}(\mathrm{c}, \mathcal{A})$, so $\mathcal{F}_{R} \mapsto \mathcal{F}_{R}^{j}$ defines a map $\phi_{i j}: t\left(\operatorname{Def}_{\mathcal{F}}\right)_{i j} \rightarrow \operatorname{Ext}_{\mathcal{A}}^{1}\left(\mathcal{F}_{j}, \mathcal{F}_{i}\right)$ that maps trivial deformations to split extensions. To construct an inverse of $\phi_{i j}$, we consider an extension $\mathcal{E}$ of $\mathcal{F}_{j}$ by $\mathcal{F}_{i}$ in $\operatorname{PreSh}(\mathrm{c}, \mathcal{A})$, and let $\mathcal{F}_{R}=\mathcal{E} \oplus \mathcal{F}_{1} \cdots \oplus \widehat{\mathcal{F}}_{i} \oplus \ldots \widehat{\oplus \mathcal{F}}_{j} \oplus \cdots \oplus \mathcal{F}_{p}$. Then $\mathcal{F}_{R}$ is a presheaf of left $\mathcal{A}$-modules on c, and it is easy to see that it defines a lifting of $\mathcal{F}$ to $R$ since $\mathcal{E}(c) \cong \mathcal{F}_{i}(c) \oplus \mathcal{F}_{j}(c)$ as $k$-linear vector spaces for any $c \in \mathrm{c}$. It follows that the assignment $\mathcal{E} \mapsto \mathcal{F}_{R}$ defines an inverse of $\phi_{i j}$.

\section{Global Hochschild cohomology}

Let $k$ be an algebraically closed field, let c be a small category, and let $\mathcal{A}$ be a presheaf of associative $k$-algebras on c. For any presheaves $\mathcal{F}, \mathcal{G}$ of left $\mathcal{A}$-modules on c, we may consider the Hochschild complex $\operatorname{HC}^{*}\left(\mathcal{A}(c), \operatorname{Hom}_{k}(\mathcal{F}(c), \mathcal{G}(c))\right)$ of $\mathcal{A}(c)$ with values in the bimodule $\operatorname{Hom}_{k}(\mathcal{F}(c), \mathcal{G}(c))$ for any object $c \in \mathrm{c}$. Unfortunately, this construction is not functorial in $c$. In this section, we consider a variation of this construction that is functorial, and use this to define a global Hochschild cohomology theory.

Let Mor c denote the category of morphisms in c defined in the following way: An object in Mor c is a morphism in c, and given objects $f: c \rightarrow c^{\prime}$ and $g: d \rightarrow d^{\prime}$ 
in Mor c, a morphism $(\alpha, \beta): f \rightarrow g$ in Mor c is a couple of morphisms $\alpha: d \rightarrow c$ and $\beta: c^{\prime} \rightarrow d^{\prime}$ in c such that $\beta f \alpha=g$. Clearly, Mor c is a small category.

Let $\mathcal{F}, \mathcal{G}$ be presheaves of left $\mathcal{A}$-modules on $\mathrm{c}$, and let $\mathcal{H o m}_{k}(\mathcal{F}, \mathcal{G})$ be the presheaf in $\operatorname{PreSh}(\operatorname{Mor} c, \underline{k})$ given by $\mathcal{H o m}_{k}(\mathcal{F}, \mathcal{G})(\phi)=\operatorname{Hom}_{k}\left(\mathcal{F}(c), \mathcal{G}\left(c^{\prime}\right)\right)$ for any morphism $\phi: c \rightarrow c^{\prime}$ in c. We define the Hochschild complex of $\mathcal{A}$ with values in $\mathcal{H o m}_{k}(\mathcal{F}, \mathcal{G})$ to be the functor $\operatorname{HC}^{*}\left(\mathcal{A}, \mathcal{H o m}{ }_{k}(\mathcal{F}, \mathcal{G})\right): \operatorname{Mor} \mathrm{c} \rightarrow \operatorname{Compl}(k)$, given by

$$
\operatorname{HC}^{p}\left(\mathcal{A}, \mathcal{H o m}_{k}(\mathcal{F}, \mathcal{G})\right)(\phi)=\operatorname{Hom}_{k}\left(\otimes_{k}^{p} \mathcal{A}(c), \operatorname{Hom}_{k}\left(\mathcal{F}(c), \mathcal{G}\left(c^{\prime}\right)\right)\right)
$$

for any morphism $\phi: c \rightarrow c^{\prime}$ in $\mathrm{c}$ and any integer $p \geq 0$, with differential given by

$$
\begin{aligned}
d^{p}(\phi)(f)\left(a_{1} \otimes \cdots \otimes a_{p+1}\right) & =a_{1} f\left(a_{2} \otimes \cdots \otimes a_{p+1}\right) \\
& +\sum_{i=1}^{p}(-1)^{i} f\left(a_{1} \otimes \cdots \otimes a_{i} a_{i+1} \otimes \cdots \otimes a_{p+1}\right) \\
& +(-1)^{p+1} f\left(a_{1} \otimes \cdots \otimes a_{p}\right) a_{p+1}
\end{aligned}
$$

for any $f \in \mathrm{HC}^{p}\left(\mathcal{A}, \mathcal{H} \operatorname{Hom}_{k}(\mathcal{F}, \mathcal{G})\right)(\phi)$ and any $a_{1}, \ldots, a_{p+1} \in \mathcal{A}(c)$. We see that $\operatorname{HC}^{*}\left(\mathcal{A}, \mathcal{H o m} m_{k}(\mathcal{F}, \mathcal{G})\right)(\phi)$ is the Hochschild complex of $\mathcal{A}(c)$ with values in the bimodule $\operatorname{Hom}_{k}\left(\mathcal{F}(c), \mathcal{G}\left(c^{\prime}\right)\right)$ for any morphism $\phi: c \rightarrow c^{\prime}$ in $\mathrm{c}$, and the definition of Mor c ensures that $\phi \mapsto \mathrm{HC}^{*}\left(\mathcal{A}, \mathcal{H} \operatorname{mom}_{k}(\mathcal{F}, \mathcal{G})\right)(\phi)$ is functorial.

Let us write $\mathcal{E} x t_{A}^{*}(\mathcal{F}, \mathcal{G}): \operatorname{Mor} \mathrm{c} \rightarrow \operatorname{Mod}(k)$ for the composition of functors given by $\phi \mapsto \mathrm{HC}^{*}\left(\mathcal{A}, \mathcal{H} \operatorname{Hom}_{k}(\mathcal{F}, \mathcal{G})\right)(\phi) \mapsto \mathrm{H}^{*}\left(\mathrm{HC}^{*}\left(\mathcal{A}, \mathcal{H} \operatorname{com}_{k}(\mathcal{F}, \mathcal{G})\right)(\phi)\right)$. We see that

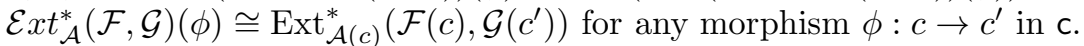

For any functor $G: \operatorname{Mor} \mathrm{c} \rightarrow \operatorname{Mod}(k)$, we may consider the resolving complex $\mathrm{D}^{*}(\mathrm{c}, G)$ in $\operatorname{Mod}(k)$ of the projective limit functor of $G$, see Laudal [15]. We recall that for any integer $p \geq 0, \mathrm{D}^{p}(\mathrm{c}, G)$ is given by

$$
\mathrm{D}^{p}(\mathrm{c}, G)=\prod_{c_{0} \rightarrow \cdots \rightarrow c_{p}} G\left(\phi_{p} \circ \cdots \circ \phi_{1}\right)
$$

where the product is taken over all $p$-tuples $\left(\phi_{1}, \ldots, \phi_{p}\right)$ of composable morphisms $\phi_{i}: c_{i-1} \rightarrow c_{i}$ in $\mathrm{c}$, and the differential $d^{p}: \mathrm{D}^{p}(\mathrm{c}, G) \rightarrow \mathrm{D}^{p+1}(\mathrm{c}, G)$ is given by

$$
\begin{aligned}
\left(d^{p} g\right)\left(\phi_{1}, \ldots, \phi_{p+1}\right) & =G\left(\phi_{1}, \mathrm{id}\right)\left(g\left(\phi_{2}, \ldots, \phi_{p+1}\right)\right) \\
& +\sum_{i=1}^{p}(-1)^{i} g\left(\phi_{1}, \ldots, \phi_{i+1} \circ \phi_{i}, \ldots, \phi_{p+1}\right) \\
& +(-1)^{p+1} G\left(\mathrm{id}, \phi_{p+1}\right)\left(g\left(\phi_{1}, \ldots, \phi_{p}\right)\right)
\end{aligned}
$$

for all $g \in \mathrm{D}^{p}(\mathrm{c}, G)$ and for all $(p+1)$-tuples $\left(\phi_{1}, \ldots, \phi_{p+1}\right)$ of composable morphisms $\phi_{i}: c_{i-1} \rightarrow c_{i}$ in c. We denote the cohomology of $\mathrm{D}^{*}(\mathrm{c}, G)$ by $\mathrm{H}^{*}(\mathrm{c}, G)$, and recall the following standard result:

Proposition 8. Let c be a small category. The resolving complex $\mathrm{D}^{*}(\mathrm{c}, G)$ has the following properties:

(1) $\mathrm{D}^{*}(\mathrm{c},-): \operatorname{PreSh}(\operatorname{Mor} \mathrm{c}, \underline{k}) \rightarrow \operatorname{Compl}(k)$ is exact,

(2) $\mathrm{H}^{p}(\mathrm{c}, G) \cong \lim ^{(p)} G$ for all $G \in \operatorname{PreSh}($ Mor $\mathrm{c}, \underline{k}$ ) and for all $p \geq 0$.

In particular, $\mathrm{H}^{*}(\mathrm{c},-): \operatorname{PreSh}(\operatorname{Mor} \mathrm{c}, \underline{k}) \rightarrow \operatorname{Mod}(k)$ is an exact $\delta$-functor.

For any functor $\mathrm{C}^{*}:$ Mor $\mathrm{c} \rightarrow \operatorname{Compl}(k)$, we may consider the double complex $\mathrm{D}^{* *}=\mathrm{D}^{*}\left(\mathrm{c}, \mathrm{C}^{*}\right)$ of vector spaces over $k$. Explicitly, have that $\mathrm{D}^{p q}=\mathrm{D}^{p}\left(\mathrm{c}, \mathrm{C}^{q}\right)$ for all integers $p, q$ with $p \geq 0$, that $d_{I}^{p q}: \mathrm{D}^{p q} \rightarrow \mathrm{D}^{p+1, q}$ is the differential $d^{p}$ in $\mathrm{D}^{*}\left(\mathrm{c}, \mathrm{C}^{q}\right)$, and that $d_{I I}^{p q}: \mathrm{D}^{p q} \rightarrow \mathrm{D}^{p, q+1}$ is the differential given by $d_{I I}^{p q}=(-1)^{p} \mathrm{D}^{p}\left(\mathrm{c}, d^{q}\right)$, 
where $d^{q}: \mathrm{C}^{q} \rightarrow \mathrm{C}^{q+1}$ is the differential in $\mathrm{C}^{*}$. Note that if $\mathrm{C}^{q}=0$ for all $q<0$, then $\mathrm{D}^{* *}$ lies in the first quadrant.

We define the global Hochschild complex of $\mathcal{A}$ with values in $\mathcal{H o m}_{k}(\mathcal{F}, \mathcal{G})$ on c to be the total complex of the double complex $\mathrm{D}^{* *}=\mathrm{D}^{*}\left(\mathrm{c}, \mathrm{HC}^{*}\left(\mathcal{A}, \mathcal{H} \operatorname{Hom}_{k}(\mathcal{F}, \mathcal{G})\right)\right)$, and denote it by $\mathrm{HC}^{*}\left(\mathrm{c}, \mathcal{A}, \mathcal{H} \mathrm{Hom}_{k}(\mathcal{F}, \mathcal{G})\right)$. Moreover, we define global Hochschild cohomology $\mathrm{HH}^{*}\left(\mathrm{c}, \mathcal{A}, \mathcal{H} \operatorname{mom}_{k}(\mathcal{F}, \mathcal{G})\right)$ of $\mathcal{A}$ with values in $\mathcal{H o m}_{k}(\mathcal{F}, \mathcal{G})$ on $\mathrm{c}$ to be the cohomology of the global Hochschild complex $\operatorname{HC}^{*}\left(\mathrm{c}, \mathcal{A}, \mathcal{H} \operatorname{Hom}_{k}(\mathcal{F}, \mathcal{G})\right)$.

Proposition 9. There is a spectral sequence for which $E_{2}^{p q}=\mathrm{H}^{p}\left(\mathrm{c}, \mathcal{E} x t_{\mathcal{A}}^{q}(\mathcal{F}, \mathcal{G})\right)$, such that $E_{\infty}=\operatorname{gr~HH}^{*}\left(\mathrm{c}, \mathcal{A}, \mathcal{H} \operatorname{Hom}_{k}(\mathcal{F}, \mathcal{G})\right)$, the associated graded vector space over $k$ with respect to a suitable filtration of $\mathrm{HH}^{*}(\mathrm{c}, \mathcal{A}, \mathcal{H o m} k(\mathcal{F}, \mathcal{G}))$.

We remark that $\mathrm{HH}^{*}\left(\mathrm{c}, \mathcal{A}, \mathcal{H} \operatorname{som}_{k}(\mathcal{F}, \mathcal{G})\right)$ can be calculated in concrete terms in many situations, using the above spectral sequence. We shall give an example of such a computation in last section of this paper.

\section{Obstruction theory FOR PREsheaves of MODUles}

Let $k$ be an algebraically closed field. For any small category c and any presheaf $\mathcal{A}$ of associative $k$-algebras on $\mathrm{c}$, we shall construct an obstruction theory for the noncommutative deformation functor $\operatorname{Def}_{\mathcal{F}}: \mathrm{a}_{p} \rightarrow$ Sets with cohomology $\left(\mathrm{HH}^{n}\left(\mathrm{c}, \mathcal{A}, \mathcal{H} \operatorname{Hom}_{k}\left(\mathcal{F}_{j}, \mathcal{F}_{i}\right)\right)\right)$ for any finite family $\mathcal{F}=\left\{\mathcal{F}_{1}, \ldots, \mathcal{F}_{p}\right\}$ of presheaves of left $\mathcal{A}$-modules on c.

Proposition 10. Let $u: R \rightarrow S$ be a small surjection in $\mathrm{a}_{p}$ with kernel $K$, and let $\mathcal{F}_{S} \in \operatorname{Def}_{\mathcal{F}}(S)$ be a deformation. Then there exists a canonical obstruction

$$
o\left(u, \mathcal{F}_{S}\right) \in\left(\mathrm{HH}^{2}\left(\mathrm{c}, \mathcal{A}, \mathcal{H o m}_{k}\left(\mathcal{F}_{j}, \mathcal{F}_{i}\right)\right) \otimes_{k} K_{i j}\right)
$$

such that $o\left(u, \mathcal{F}_{S}\right)=0$ if and only if there exists a deformation $\mathcal{F}_{R} \in \operatorname{Def}_{\mathcal{F}}(R)$ lifting $\mathcal{F}_{S}$ to $R$. Moreover, if o $\left(u, \mathcal{F}_{S}\right)=0$, then there is a transitive and effective action of $\left(\mathrm{HH}^{1}\left(\mathrm{c}, \mathcal{A}, \mathcal{H o m}_{k}\left(\mathcal{F}_{j}, \mathcal{F}_{i}\right)\right) \otimes_{k} K_{i j}\right)$ on the set of liftings of $\mathcal{F}_{S}$ to $R$.

Proof. Let $\mathcal{F}_{S} \in \operatorname{Def}_{\mathcal{F}}(S)$ be given. By lemma 6, this deformation corresponds to the following data: A $k$-algebra homomorphism $L^{S}(c): \mathcal{A}(c) \rightarrow Q^{S}(c)$ for each object $c \in \mathrm{c}$ and an element $L^{S}(\phi) \in Q^{S}\left(c, c^{\prime}\right)$ for each morphism $\phi: c \rightarrow c^{\prime}$ in c, such that the conditions of lemma 6 are satisfied. Moreover, to lift $\mathcal{F}_{S}$ to $R$ is the same as to lift these data to $R$.

Choose a $k$-linear section $\sigma: S \rightarrow R$ such that $\sigma\left(I(S)_{i j}\right) \subseteq I(R)_{i j}$ and $\sigma\left(e_{i}\right)=e_{i}$ for $1 \leq i, j \leq p$. Clearly, $\sigma$ induces a $k$-linear map $Q^{S}\left(c, c^{\prime}\right) \rightarrow Q^{R}\left(c, c^{\prime}\right)$ for all $c, c^{\prime}$ in c, which we shall denote by $Q\left(\sigma, c, c^{\prime}\right)$. We define $L^{R}(c)=Q(\sigma, c, c) \circ L^{S}(c)$ for all objects $c \in \mathrm{c}$ and $L^{R}(\phi)=Q\left(\sigma, c, c^{\prime}\right)\left(L^{S}\left(c, c^{\prime}\right)\right)$ for all morphisms $\phi: c \rightarrow c^{\prime}$ in c. Then $L^{R}(c): \mathcal{A}(c) \rightarrow Q^{R}(c)$ is a $k$-linear map which lifts $L^{S}(c)$ to $R$ for all $c \in \mathrm{c}$, and we define the obstruction

$$
o(0,2)(c): \mathcal{A}(c) \otimes_{k} \mathcal{A}(c) \rightarrow Q^{K}(c)
$$

by $o(0,2)(c)(a \otimes b)=L^{R}(c)(a b)-L^{R}(c)(a) L^{R}(c)(b)$ for all $a, b \in \mathcal{A}(c)$. It is clear that $L^{R}(c)$ is a $k$-algebra homomorphism if and only if $o(0,2)(c)=0$. Moreover, $L^{R}(\phi)$ lifts $L^{S}(\phi)$ to $R$ for all morphisms $\phi: c \rightarrow c^{\prime}$ in c, and we define the obstruction

$$
o(1,1)(\phi): \mathcal{A}(c) \rightarrow Q^{K}\left(c, c^{\prime}\right)
$$


by $o(1,1)(\phi)(a)=L^{R}(\phi) \circ L^{R}(c)(a)-L^{R}\left(c^{\prime}\right)(\mathcal{A}(\phi)(a)) \circ L^{R}(\phi)$ for all $a \in \mathcal{A}(c)$. It is clear that $L^{R}(\phi)$ is $\mathcal{A}(\phi)$-linear if and only if $o(1,1)(\phi)=0$. Finally, we define the obstruction

$$
o(2,0)\left(\phi, \phi^{\prime}\right) \in Q^{K}\left(c, c^{\prime \prime}\right)
$$

by $o(2,0)\left(\phi, \phi^{\prime}\right)=L^{R}\left(\phi^{\prime}\right) L^{R}(\phi)-L^{R}\left(\phi^{\prime} \circ \phi\right)$ for all morphisms $\phi: c \rightarrow c^{\prime}$ and $\phi^{\prime}: c^{\prime} \rightarrow c^{\prime \prime}$ in c. It is clear that $L^{R}$ satisfies the cocycle condition if and only if $o(2,0)=0$.

We see that $o=(o(0,2), o(1,1), o(2,0))$ is a 2-cochain in global Hochschild complex $\left(\mathrm{HC}^{n}\left(\mathrm{c}, \mathcal{A}, \mathcal{H} \operatorname{Hom}_{k}\left(\mathcal{F}_{j}, \mathcal{F}_{i}\right)\right) \otimes_{k} K_{i j}\right)$. A calculation shows that $o$ is a 2-cocycle, and that its cohomology class $o\left(u, \mathcal{F}_{S}\right) \in\left(\mathrm{HH}^{2}\left(\mathrm{c}, \mathcal{A}, \mathcal{H} \operatorname{Hom}_{k}\left(\mathcal{F}_{j}, \mathcal{F}_{i}\right)\right) \otimes_{k} K_{i j}\right)$ is independent of the choice of $L^{R}(c)$ and $L^{R}(\phi)$. It is clear that if there is a lifting of $\mathcal{F}_{S}$ to $R$, we may choose $L^{R}(c)$ and $L^{R}(\phi)$ such that $o=0$, hence $o\left(u, \mathcal{F}_{S}\right)=0$. Conversely, assume that $o\left(u, \mathcal{F}_{S}\right)=0$. Then there exists a 1-cochain of the form $(\epsilon, \Delta)$ with $\epsilon \in \mathrm{D}^{01}$ and $\Delta \in \mathrm{D}^{10}$ such that $d(\epsilon, \Delta)=o$. Let $L^{\prime}(c)=L^{R}(c)+\epsilon(c)$ and $L^{\prime}(\phi)=L^{R}(\phi)+\Delta(\phi)$. Then $L^{\prime}(c)$ is another lifting of $L^{S}(c)$ to $R, L^{\prime}(\phi)$ is another lifting of $L^{S}(\phi)$ to $R$, and essentially the same calculation as above shows that the corresponding 2-cocycle $o^{\prime}=0$. Hence there is a lifting of $\mathcal{F}_{S}$ to $R$, and this proves the first part of the proposition.

For the second part, assume that $\mathcal{F}_{R}$ is a lifting of $\mathcal{F}$ to $R$. Then $\mathcal{F}_{R}$ is defined by liftings $L^{R}(c)$ and $L^{R}(\phi)$ to $R$ such that the corresponding 2-cocycle $o=0$. Let us consider a 1-cochain $(\epsilon, \Delta)$, and consider the new liftings $L^{\prime}(c)=L^{R}(c)+\epsilon(c)$ and $L^{\prime}(\phi)=L^{R}(\phi)+\Delta(\phi)$. From the previous calculations, it is clear that the new 2 -cocycle $o^{\prime}=0$ if and only if $(\epsilon, \Delta)$ is a 1-cocycle. Moreover, if this is the case, the lifting $\mathcal{F}_{R}^{\prime}$ defined by $L^{\prime}(c)$ and $L^{\prime}(\phi)$ is equivalent to $\mathcal{F}_{R}$ if and only if $(\epsilon, \Delta)$ is a 1-coboundary, since an equivalence between $\mathcal{F}_{R}$ and $\mathcal{F}_{R}^{\prime}$ must have the form id $+\pi$ for some 0-cochain $\pi$ with $d(\pi)=(\epsilon, \Delta)$.

We see that the obstruction $o\left(u, \mathcal{F}_{S}\right)$ is functorial, so it defines an obstruction theory for the noncommutative deformation functor $\operatorname{Def}_{\mathcal{F}}: a_{p} \rightarrow$ Sets by definition. If the condition

$$
\operatorname{dim}_{k} \mathrm{HH}^{n}\left(\mathrm{c}, \mathcal{A}, \mathcal{H o m}_{k}\left(\mathcal{F}_{j}, \mathcal{F}_{i}\right)\right)<\infty \text { for } 1 \leq i, j \leq p, n=1,2,
$$

holds, it follows that $\operatorname{Def}_{\mathcal{F}}$ has an obstruction theory with finite dimensional coho$\operatorname{mology}\left(\mathrm{HH}^{n}\left(\mathrm{c}, \mathcal{A}, \mathcal{H} \operatorname{Hom}_{k}\left(\mathcal{F}_{j}, \mathcal{F}_{i}\right)\right)\right)$.

Theorem 11. Let c be a small category, let $\mathcal{A}$ be a presheaf of $k$-algebras on $\mathrm{c}$, and let $\mathcal{F}=\left\{\mathcal{F}_{1}, \ldots, \mathcal{F}_{p}\right\}$ be a finite family of presheaves of left $\mathcal{A}$-modules on $\mathrm{c}$. If condition (4) holds, then the noncommutative deformation functor $\operatorname{Def}_{\mathcal{F}}: \mathrm{a}_{p} \rightarrow$ Sets of $\mathcal{F}$ in $\operatorname{PreSh}(\mathrm{c}, \mathcal{A})$ has a pro-representing hull $\mathrm{H}\left(\operatorname{Def}_{\mathcal{F}}\right)$, completely determined by the $k$-linear spaces $\mathrm{HH}^{n}\left(\mathrm{c}, \mathcal{A}, \mathcal{H}_{\text {om }}\left(\mathcal{F}_{j}, \mathcal{F}_{i}\right)\right)$ for $1 \leq i, j \leq p, n=1,2$, together with some generalized Massey products on them.

Corollary 12. Let $\mathrm{c}$ be a small category, let $\mathcal{A}$ be a presheaf of $k$-algebras on $\mathrm{c}$, and let $\mathcal{F}=\left\{\mathcal{F}_{1}, \ldots, \mathcal{F}_{p}\right\}$ be a finite family of presheaves of left $\mathcal{A}$-modules on $\mathrm{c}$. If (4) holds, then $\mathrm{HH}^{1}\left(\mathrm{c}, \mathcal{A}, \mathcal{H}_{k}\left(\mathcal{F}_{j}, \mathcal{F}_{i}\right)\right) \cong t\left(\operatorname{Def}_{\mathcal{F}}\right)_{i j} \cong \operatorname{Ext}_{\mathcal{A}}^{1}\left(\mathcal{F}_{j}, \mathcal{F}_{i}\right)$ for $1 \leq i, j \leq p$.

\section{Deformations of Sheaves of modules}

Let $k$ be an algebraically closed field, and let $(X, \mathcal{A})$ be a ringed space over $k$, i.e. a topological space $X$ together with a sheaf $\mathcal{A}$ of associative $k$-algebras on $X$. 
The category $\operatorname{Sh}(X, \mathcal{A})$ of sheaves of left $\mathcal{A}$-modules on $X$ is an Abelian $k$-category, and we shall consider deformations in this category.

Let $\mathrm{c}(X)$ be the category defined in the following way: An object in $\mathrm{c}(X)$ is an open subset $U \subseteq X$, and given objects $U, V \in \mathrm{c}(X)$, a morphism from $U$ to $V$ in $\mathrm{c}(X)$ is an (opposite) inclusion $U \supseteq V$. Then $\mathrm{c}(X)$ is a small category, and we may consider $\operatorname{Sh}(X, \mathcal{A})$ as the full subcategory of $\operatorname{PreSh}(\mathrm{c}(X), \mathcal{A})$ consisting of exactly those presheaves of left $\mathcal{A}$-modules on $\mathrm{c}(X)$ that satisfy the sheaf axioms. It is wellknown that this subcategory is closed under direct sums, kernels and extensions, but not under cokernels.

Let $\mathcal{F}=\left\{\mathcal{F}_{1}, \ldots, \mathcal{F}_{p}\right\}$ be a finite family of sheaves of left $\mathcal{A}$-modules on $X$. Since $\operatorname{Sh}(X, \mathcal{A})$ is an Abelian $k$-category and a full subcategory of $\operatorname{PreSh}(\mathrm{c}(X), \mathcal{A})$, we may consider the noncommutative deformation functor $\operatorname{Def}_{\mathcal{F}}^{s h}: a_{p} \rightarrow$ Sets of $\mathcal{F}$ as a family of sheaves, defined by $\operatorname{Def}_{\mathcal{F}}^{s h}=\operatorname{Def}_{\mathcal{F}}^{\mathrm{A}}$ with $\mathrm{A}=\operatorname{Sh}(X, \mathcal{A})$. We may also consider the noncommutative deformation functor $\operatorname{Def}_{\mathcal{F}}: \mathrm{a}_{p} \rightarrow$ Sets of $\mathcal{F}$ as a family of presheaves, given by $\operatorname{Def}_{\mathcal{F}}=\operatorname{Def}_{\mathcal{F}}^{\mathrm{A}}$ with $\mathrm{A}=\operatorname{PreSh}(\mathrm{c}(X), \mathcal{A})$, and we remark that the natural forgetful functor $\operatorname{Sh}(X, \mathcal{A}) \rightarrow \operatorname{PreSh}(\mathrm{c}(X), \mathcal{A})$ induces an isomorphism of noncommutative deformation functors $\operatorname{Def}_{\mathcal{F}}^{s h} \stackrel{\sim}{\rightarrow} \operatorname{Def}_{\mathcal{F}}$. However, this observation is not very useful for computational purposes, since the category $\mathrm{c}(X)$ is usually too big to allow for effective computations.

Finally, we remark that $t\left(\operatorname{Def}_{\mathcal{F}}^{s h}\right)_{i j} \cong t\left(\operatorname{Def}_{\mathcal{F}}\right)_{i j}$ since $\operatorname{Def}_{\mathcal{F}}^{s h} \cong \operatorname{Def}_{\mathcal{F}}$, hence $t\left(\operatorname{Def}_{\mathcal{F}}^{s h}\right)_{i j} \cong \operatorname{HH}^{1}\left(\mathrm{c}(X), \mathcal{A}, \mathcal{H o m}_{k}\left(\mathcal{F}_{j}, \mathcal{F}_{i}\right)\right) \cong \operatorname{Ext}_{\mathcal{A}}^{1}\left(\mathcal{F}_{j}, \mathcal{F}_{i}\right)$. It does not matter if we consider $\operatorname{Ext}_{\mathcal{A}}^{1}\left(\mathcal{F}_{j}, \mathcal{F}_{i}\right)$ as extensions in $\operatorname{Sh}(X, \mathcal{A})$ or $\operatorname{PreSh}(\mathrm{c}(X), \mathcal{A})$, since $\operatorname{Sh}(X, \mathcal{A})$ is closed under extensions in $\operatorname{PreSh}(\mathrm{c}(X), \mathcal{A})$.

\section{Deformations of QUASI-COHERENT SheAVES OF MODUleS}

Let $k$ be an algebraically closed field, and let $(X, \mathcal{A})$ be a ringed space over $k$. We recall that a sheaf $\mathcal{F} \in \operatorname{Sh}(X, \mathcal{A})$ of left $\mathcal{A}$-modules on $X$ is quasi-coherent if for every point $x \in X$, there exists an open neighbourhood $U \subseteq X$ of $x$, free sheaves $\mathcal{L}_{0}, \mathcal{L}_{1}$ of left $\left.\mathcal{A}\right|_{U}$-modules on $U$, and an exact sequence

$$
\left.0 \leftarrow \mathcal{F}\right|_{U} \leftarrow \mathcal{L}_{0} \leftarrow \mathcal{L}_{1}
$$

of sheaves of left $\left.\mathcal{A}\right|_{U}$-modules on $U$. The category $\mathrm{Q} \operatorname{Coh}(\mathcal{A})$ of quasi-coherent sheaves of left $\mathcal{A}$-modules on $X$ is the full subcategory of $\operatorname{Sh}(X, \mathcal{A})$ consisting of quasi-coherent sheaves.

The full subcategory $\mathrm{Q} \operatorname{Coh}(\mathcal{A}) \subseteq \operatorname{Sh}(X, \mathcal{A})$ is closed under finite direct sums, but it is not clear if $\mathrm{Q} \operatorname{Coh}(\mathcal{A})$ is closed under kernels and cokernels in general. Hence $\mathrm{QCoh}(\mathcal{A})$ is an additive but not necessarily an Abelian $k$-category. In this section, we give sufficient conditions for $\mathrm{Q} \operatorname{Coh}(\mathcal{A})$ to be an exact Abelian subcategory of $\operatorname{Sh}(X, \mathcal{A})$, and consider deformations in the category $\mathrm{Q} \operatorname{Coh}(\mathcal{A})$ in these cases.

Let us consider the global sections functor $\Gamma(X,-): \operatorname{Sh}(X, \mathcal{A}) \rightarrow \operatorname{Mod}(A)$, where we write $A=\Gamma(X, \mathcal{A})$. This functor is left exact, and we denote its right derived functors by $\mathrm{H}^{*}(X,-)=R^{*} \Gamma(X,-)$. We say that $X$ is $\mathcal{A}$-affine if the following conditions hold:

(1) $\Gamma(X,-)$ induces an equivalence of categories $\mathrm{Q} \operatorname{Coh}(\mathcal{A}) \rightarrow \operatorname{Mod}(A)$,

(2) $\mathrm{H}^{n}(X, \mathcal{F})=0$ for all $\mathcal{F} \in \mathrm{Q} \operatorname{Coh}(\mathcal{A})$ and for all integers $n \geq 1$.

Moreover, we say that an open subset $U \subseteq X$ is $\mathcal{A}$-affine if $U$ is $\left.\mathcal{A}\right|_{U \text {-affine, and }}$ that an open cover $\mathrm{U}$ of $X$ is $\mathcal{A}$-affine if $U$ is $\mathcal{A}$-affine for any $U \in \mathrm{U}$. 
We say that a full subcategory $\mathrm{C}$ of an Abelian $k$-category $\mathrm{A}$ is thick if $\mathrm{C} \subseteq \mathrm{A}$ is an exact Abelian subcategory that is closed under extensions, and recall that

(1) If $X_{1} \rightarrow X_{2} \rightarrow Y \rightarrow X_{3} \rightarrow X_{4}$ is an exact sequence in A with $X_{i} \in \mathrm{C}$ for $1 \leq i \leq 4$, then $Y \in \mathrm{C}$.

is a necessary and sufficient condition for $\mathrm{C} \subseteq \mathrm{A}$ to be a thick subcategory.

Proposition 13. If $X$ has an $\mathcal{A}$-affine open cover, then $\mathrm{QCoh}(\mathcal{A}) \subseteq \operatorname{Sh}(X, \mathcal{A})$ is a thick subcategory.

Proof. Using the condition (1) above, we see that QCoh $(\mathcal{A})$ is a thick subcategory of $\operatorname{Sh}(X, \mathcal{A})$ if and only this holds locally. We may therefore assume that $X$ is $\mathcal{A}$-affine. This implies that $\mathrm{Q} \operatorname{Coh}(\mathcal{A})$ has kernels and cokernels, since this holds for $\operatorname{Mod}(A)$. We must show that $\mathrm{Q} \operatorname{Coh}(\mathcal{A})$ is closed under extensions. Let $0 \rightarrow \mathcal{F} \rightarrow \mathcal{G} \rightarrow \mathcal{H} \rightarrow 0$ be an exact sequence in $\operatorname{Sh}(X, \mathcal{A})$ with $\mathcal{F}, \mathcal{H}$ in $\mathrm{Q} \operatorname{Coh}(\mathcal{A})$. Then $\mathrm{H}^{1}(X, \mathcal{F})=0$, and $\Gamma(X,-): \operatorname{Sh}(X, \mathcal{A}) \rightarrow \operatorname{Mod}(A)$ is right adjoint to $\mathrm{qc}_{X}: \operatorname{Mod}(A) \rightarrow \mathrm{Q} \operatorname{Coh}(\mathcal{A})$, the quasi-inverse of $\Gamma(X,-)$ restricted to $\mathrm{Q} \operatorname{Coh}(\mathcal{A})$. So there is a commutative diagram

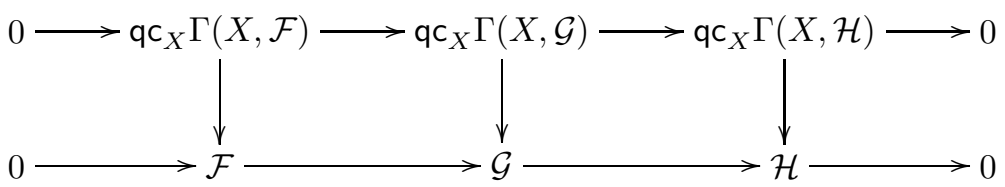

in $\operatorname{Sh}(X, \mathcal{A})$ with exact rows. The left and right vertical arrows are isomorphisms, so the middle vertical arrow is an isomorphism as well.

Let $\mathrm{U}$ be an $\mathcal{A}$-affine open cover of $X$, viewed as a small subcategory of $\mathrm{c}(X)$, and let $\pi: \mathrm{QCoh}(\mathcal{A}) \rightarrow \operatorname{PreSh}(\mathrm{U}, \mathcal{A})$ be the natural forgetful functor. For any finite family $\mathcal{F}=\left\{\mathcal{F}_{1}, \ldots, \mathcal{F}_{p}\right\}$ of quasi-coherent sheaves of left $\mathcal{A}$-modules on $X$, we may consider the noncommutative deformation functor $\operatorname{Def}_{\mathcal{F}}^{q c}: a_{p} \rightarrow$ Sets of $\mathcal{F}$ as a family of quasi-coherent sheaves, defined by $\operatorname{Def}_{\mathcal{F}}^{q c}=\operatorname{Def}_{\mathcal{F}}^{\mathrm{A}}$ with $\mathrm{A}=\mathrm{Q} \operatorname{Coh}(\mathcal{A})$. We may also consider the noncommutative deformation functor $\operatorname{Def}_{\mathcal{F}}^{U}: \mathrm{a}_{p} \rightarrow$ Sets of $\mathcal{F}$ as a family of presheaves on $\mathrm{U}$, defined by $\operatorname{Def}_{\mathcal{F}}^{U}=\operatorname{Def}_{\mathcal{F}}^{\mathrm{A}}$ with $\mathrm{A}=\operatorname{PreSh}(\mathrm{U}, \mathcal{A})$. We remark that $\pi$ induces a morphism $\operatorname{Def}_{\mathcal{F}}^{q c} \rightarrow \operatorname{Def}_{\mathcal{F}}^{U}$ of noncommutative deformation functors, but not necessarily an isomorphism.

An open cover $\mathrm{U}$ of $X$ is good if any finite intersection $V=U_{1} \cap U_{2} \cap \cdots \cap U_{r}$ with $U_{i} \in \mathrm{U}$ for $1 \leq i \leq r$ can be covered by open subsets $W \subseteq V$ with $W \in \mathrm{U}$. In particular, any open cover closed under finite intersections is good.

Proposition 14. If $\bigcup$ is a good $\mathcal{A}$-affine open cover of $X$, then the forgetful functor $\pi: \mathrm{QCoh}(\mathcal{A}) \rightarrow \operatorname{PreSh}(\mathrm{U}, \mathcal{A})$ induces an isomorphism $\operatorname{Def}_{\mathcal{F}}^{q c} \rightarrow \operatorname{Def}_{\mathcal{F}}^{U}$ of deformation functors for any finite family $\mathcal{F}$ of quasi-coherent left $\mathcal{A}$-modules on $X$.

Proof. Clearly, $\pi$ induces a morphism of noncommutative deformation functors, and it is enough to show that the induced map of sets $\pi_{R}^{*}: \operatorname{Def}_{\mathcal{F}}^{q c}(R) \rightarrow \operatorname{Def}_{\mathcal{F}}^{\mathrm{U}}(R)$ is a bijection for any $R \in \mathrm{a}_{p}$. If $X$ is $\mathcal{A}$-affine and $\mathrm{U}=\{X\}$, then $\operatorname{PreSh}(\mathrm{U}, \mathcal{A})$ is naturally equivalent to $\operatorname{Mod}(A)$, so $\pi: \mathrm{QCoh}(\mathcal{A}) \rightarrow \operatorname{PreSh}(\mathrm{U}, \mathcal{A})$ is an equivalence of categories, and this implies that $\pi_{R}$ is a bijection for any $R \in \mathrm{a}_{p}$. In the general case, let $\mathcal{F}_{R} \in \operatorname{Def}_{\mathcal{F}}^{U}(R)$. Then $\mathcal{F}_{R}(U)$ is a deformation of the family $\left\{\mathcal{F}_{1}(U), \ldots, \mathcal{F}_{p}(U)\right\}$ in $\operatorname{Mod}(\mathcal{A}(U))$ to $R$ for any $U \in \mathrm{U}$. By the result in the $\mathcal{A}$-affine case, we can find a deformation $\mathcal{F}_{R}^{U}$ of the family $\left\{\left.\mathcal{F}_{1}\right|_{U}, \ldots,\left.\mathcal{F}_{p}\right|_{U}\right\}$ in $\mathrm{QCoh}\left(\left.\mathcal{A}\right|_{U}\right)$ to $R$ that is compatible with $\mathcal{F}_{R}(U)$. We remark that if $V \subseteq U$ is an inclusion in $\mathrm{U}$, then there 
is a natural isomorphism $\left.\mathcal{F}_{R}^{U}\right|_{V} \rightarrow \mathcal{F}_{R}^{V}$ of sheaves of left $\mathcal{A}$-modules on $V$, since $\mathcal{F}$ is a family of quasi-coherent sheaves of $\mathcal{A}$-modules on $X$. We must glue the local deformations $\mathcal{F}_{R}^{U}$ to a deformation $\mathcal{F}_{R}$ of the family $\mathcal{F}$ to $R$ in $\mathrm{Q} \operatorname{Coh}(\mathcal{A})$, and this is clearly possible since $\mathrm{U}$ is a good open cover of $X$.

Theorem 15. Let $(X, \mathcal{A})$ be a ringed space over $k$, let $\mathrm{U}$ be a good $\mathcal{A}$-affine open cover of $X$, and let $\mathcal{F}=\left\{\mathcal{F}_{1}, \ldots, \mathcal{F}_{p}\right\}$ be a finite family of quasi-coherent left $\mathcal{A}$ modules on $X$. If $\operatorname{dim}_{k} \mathrm{HH}^{n}\left(\mathrm{U}, \mathcal{A}, \mathcal{H}\right.$ om $\left._{k}\left(\mathcal{F}_{j}, \mathcal{F}_{i}\right)\right)<\infty$ for $1 \leq i, j \leq p, n=1,2$, then the noncommutative deformation functor $\operatorname{Def}_{\mathcal{F}}^{q c}: \mathrm{a}_{p} \rightarrow$ Sets of $\mathcal{F}$ in $\mathrm{QCoh}(\mathcal{A})$ has a pro-representing hull $\mathrm{H}\left(\operatorname{Def}_{\mathcal{F}}^{q c}\right)$, completely determined by the $k$-linear spaces $\mathrm{HH}^{n}\left(\mathrm{U}, \mathcal{A}, \mathcal{H o m}_{k}\left(\mathcal{F}_{j}, \mathcal{F}_{i}\right)\right)$ for $1 \leq i, j \leq p, n=1,2$, together with some generalized Massey products on them.

We remark that if the conditions of theorem 15 holds, then $t\left(\operatorname{Def}_{\mathcal{F}}^{q c}\right)_{i j} \cong t\left(\operatorname{Def}_{\mathcal{F}}^{U}\right)_{i j}$ since $\operatorname{Def}_{\mathcal{F}}^{q c} \cong \operatorname{Def}_{\mathcal{F}}^{U}$, hence $t\left(\operatorname{Def}_{\mathcal{F}}^{q c}\right)_{i j} \cong \operatorname{HH}^{1}\left(\mathrm{U}, \mathcal{A}, \mathcal{H}\right.$ om $\left._{k}\left(\mathcal{F}_{j}, \mathcal{F}_{i}\right)\right) \cong \operatorname{Ext}_{\mathcal{A}}^{1}\left(\mathcal{F}_{j}, \mathcal{F}_{i}\right)$. It does not matter if we consider $\operatorname{Ext}_{\mathcal{A}}^{1}\left(\mathcal{F}_{j}, \mathcal{F}_{i}\right)$ as extensions in $\operatorname{PreSh}(\mathrm{U}, \mathcal{A})$ or in $\mathrm{QCoh}(\mathcal{A})$, since the forgetful functor $\mathrm{Q} \operatorname{Coh}(\mathcal{A}) \rightarrow \operatorname{PreSh}(\mathrm{U}, \mathcal{A})$ preserves extensions by the argument in the proof of proposition 14

\section{QUASI-COHERENT RINGED SCHEMES}

Let $k$ be an algebraically closed field. We shall consider some important examples of ringed spaces $(X, \mathcal{A})$ over $k$ such that $X$ has a good $\mathcal{A}$-affine open cover $\mathrm{U}$. It is often possible to choose $\mathrm{U}$ to be a finite cover, and this is important for effective computations of noncommutative deformations using presheaf methods.

Example 1. Let $\left(X, \mathcal{O}_{X}\right)$ be a scheme over $k$. If $U \subseteq X$ is an open affine subscheme of $X$, then $U$ is $\mathcal{O}_{X}$-affine by Hartshorne [12, corollary II.5.5 and Grothendieck [7, theorem 1.3.1. Hence any open affine cover of $X$ is an $\mathcal{O}_{X}$-affine open cover. If $X$ is separated over $k$, then any finite intersection of open affine subschemes of $X$ is affine. Hence if $X$ is quasi-compact and separated over $k$, then there is a finite

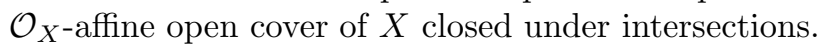

Example 2. A ringed scheme over $k$ is a ringed space $(X, \mathcal{A})$ over $k$ defined by a scheme $\left(X, \mathcal{O}_{X}\right)$ over $k$ and a morphism $i: \mathcal{O}_{X} \rightarrow \mathcal{A}$ of sheaves of associative $k$-algebras on $X$. A quasi-coherent ringed scheme over $k$ is a ringed scheme $(X, \mathcal{A})$ over $k$ such that $\mathcal{A}$ is quasi-coherent as a left and right $\mathcal{O}_{X}$-module. The notion of quasi-coherent ringed schemes was introduced in Yekutieli, Zhang [19, and the following result follows from Yekutieli, Zhang [19], corollary 5.13:

Lemma 16. A ringed scheme $(X, \mathcal{A})$ over $k$ is quasi-coherent if and only if the morphism $\mathcal{A}(U) \rightarrow \mathcal{A}(D(f))$ is a ring of fractions with respect to $S=\left\{f^{n}: n \geq 0\right\}$ for any open affine subscheme $U \subseteq X$ and any $f \in \mathcal{O}_{X}(U)$.

For any quasi-coherent ringed scheme $(X, \mathcal{A})$ over $k$, a left $\mathcal{A}$-module $\mathcal{F}$ is quasicoherent if and only if it is quasi-coherent as a left $\mathcal{O}_{X}$-module. This follows from Grothendieck [6], proposition 9.6.1 when $\mathcal{A}$ is a sheaf of commutative rings on $X$, and the proof can easily be extended to the noncommutative case.

Lemma 17. Let $(X, \mathcal{A})$ be a quasi-coherent ringed scheme over $k$. If $U \subseteq X$ is an open affine subscheme of $X$, then $U$ is $\mathcal{A}$-affine. 
Proof. Write $A=\mathcal{A}(U)$, and consider $\Gamma(U,-): \mathrm{QCoh}\left(\left.\mathcal{A}\right|_{U}\right) \rightarrow \operatorname{Mod}(A)$. We claim that $\Gamma(U,-)$ is an equivalence of categories. By the comments preceding the lemma, $\mathrm{Q} \operatorname{Coh}\left(\left.\mathcal{A}\right|_{U}\right)$ can be considered as a subcategory of $\mathrm{Q} \operatorname{Coh}\left(\mathcal{O}_{U}\right)$, and $\Gamma(U,-): \mathrm{Q} \operatorname{Coh}\left(\mathcal{O}_{U}\right) \rightarrow \operatorname{Mod}(O)$ is an equivalence of categories with $O=\mathcal{O}_{X}(U)$. So the claim follows from lemma 16. Finally, $\mathrm{H}^{n}(U, \mathcal{F})=0$ for any integer $n \geq 1$ and any $\mathcal{F} \in \mathrm{Q} \operatorname{Coh}\left(\left.\mathcal{A}\right|_{U}\right)$ by the above comments.

Let $(X, \mathcal{A})$ be a quasi-coherent ringed scheme over $k$. Then any open affine cover of $X$ is an $\mathcal{A}$-affine open cover of $X$. If $X$ is quasi-compact and separated over $k$, then there is a finite $\mathcal{A}$-affine open cover of $X$ closed under intersections.

Example 3 . Let $(X, \mathcal{A})$ be a quasi-coherent ringed scheme over $k$, and assume that $\operatorname{char}(k)=0$. We say that $\mathcal{A}$ is a $D$-algebra, and that $(X, \mathcal{A})$ is a $D$-scheme, if the following condition holds: For any open subset $U \subseteq X$ and for any section $a \in \mathcal{A}(U)$, there exists an integer $n \geq 0$ (depending on $a$ ) such that

$$
\left[\ldots\left[\left[a, f_{1}\right], f_{2}\right] \ldots, f_{n}\right]=0
$$

for all sections $f_{1}, \ldots, f_{n} \in \mathcal{O}_{X}(U)$, where $[a, f]=a f-f a$ is the usual commutator for all $a \in \mathcal{A}(U), f \in \mathcal{O}_{X}(U)$. The notion of D-schemes was considered in Beilinson, Bernstein 1, and most quasi-coherent ringed schemes that appear naturally are D-schemes. We give some important examples of D-schemes below.

Example 4. Let $\left(X, \mathcal{O}_{X}\right)$ be a scheme over $k$, and assume that $\operatorname{char}(k)=0$. For any sheaf $\mathcal{F}$ of $\mathcal{O}_{X}$-modules, we denote by $\operatorname{Diff}(\mathcal{F})$ the sheaf of $k$-linear differential operators on $\mathcal{F}$, see Grothendieck [8], section 16.8. By definition, Diff $(\mathcal{F})$ is a sheaf of associative $k$-algebras on $X$, equipped with a morphism $i: \mathcal{O}_{X} \rightarrow \operatorname{Diff}(\mathcal{F})$ of sheaves of rings, and $\operatorname{Diff}(\mathcal{F})$ is clearly a D-algebra on $X$ if and only if $\operatorname{Diff}(\mathcal{F})$ is quasi-coherent as a left and right $\mathcal{O}_{X}$-module. By Beilinson, Bernstein [1], example 1.1.6, this is the case if $\mathcal{F}$ is a coherent $\mathcal{O}_{X}$-module.

Let $X$ be locally Noetherian, and consider the sheaf $\mathcal{D}_{X}=\operatorname{Diff}\left(\mathcal{O}_{\mathcal{X}}\right)$ of $k$-linear differential operators on $X$. Since $\mathcal{O}_{X}$ is a coherent sheaf of rings, it follows that $\mathcal{D}_{X}$ is a D-algebra on $X$.

We remark that there are some examples of schemes over $k$ that are $\mathcal{D}_{X}$-affine but not affine. For instance, this holds for the projective space $X=\mathbf{P}^{n}$ for all integers $n \geq 1$, see Beilinson, Bernstein [2]. It also holds for the weighted projective space $X=\mathbf{P}\left(a_{1}, \ldots, a_{n}\right)$, see Van den Bergh [18].

Example 5. Let $\left(X, \mathcal{O}_{X}\right)$ be a separated scheme of finite type over $k$, and assume that $\operatorname{char}(k)=0$. A Lie algebroid of $X$ is a quasi-coherent $\mathcal{O}_{X}$-module $\mathbf{g}$ with a $k$-Lie algebra structure, together with a morphism $\tau: \mathbf{g} \rightarrow \operatorname{Der}_{k}\left(\mathcal{O}_{X}\right)$ of sheaves of $\mathcal{O}_{X}$-modules and of $k$-Lie algebras, such that

$$
[g, f \cdot h]=f[g, h]+\tau_{g}(f) \cdot h
$$

for any open subset $U \subseteq X$ and any sections $g, h \in \mathbf{g}(U), f \in \mathcal{O}_{X}(U)$. The notion of Lie algebroids in algebraic geometry was considered in Beilinson, Bernstein [1].

For any sheaf $\mathcal{F}$ of $\mathcal{O}_{X}$-modules, an integrable $\mathbf{g}$-connection on $\mathcal{F}$ is a morphism $\nabla: \mathbf{g} \rightarrow \mathcal{E} n d_{k}(\mathcal{F})$ of sheaves of $\mathcal{O}_{X}$-modules and of $k$-Lie algebras such that

$$
\nabla_{U}(g)(f m)=f \nabla_{U}(g)(m)+g(f) m
$$

for any open subset $U \subseteq X$ and any sections $f \in \mathcal{O}_{X}(U), g \in \mathbf{g}(U), m \in \mathcal{F}(U)$. The quasi-coherent sheaves of $\mathcal{O}_{X}$-modules with integrable g-connections form an 
Abelian $k$-category, and there is a universal enveloping D-algebra $\mathrm{U}(\mathbf{g})$ of $\mathbf{g}$ such that this category is equivalent to $\mathrm{QCoh}(\mathrm{U}(\mathbf{g}))$, see Beilinson, Bernstein [1. In particular, $(X, \mathrm{U}(\mathbf{g}))$ is a $\mathrm{D}$-scheme.

The tangent sheaf $\theta_{X}=\operatorname{Der}_{k}\left(\mathcal{O}_{X}\right)$ of $X$ is a Lie algebroid of $X$ in a natural way, and $\mathrm{U}\left(\theta_{X}\right)$ is a subsheaf of the sheaf $\mathcal{D}_{X}$ of $k$-linear differential operators on $X$. If $X$ is a smooth irreducible quasi-projective variety over $k$, then $\mathrm{U}\left(\theta_{X}\right)=\mathcal{D}_{X}$.

\section{Calculations For D-Modules on Elliptic Curves}

Let $k$ be an algebraically closed field of characteristic 0 , and let $X$ be a smooth irreducible variety over $k$ of dimension $d$. Then the sheaf $\mathcal{D}_{X}$ of $k$-linear differential operators on $X$ is a D-algebra on $X$. We consider the noncommutative deformations of $\mathcal{O}_{X}$ as a quasi-coherent left $\mathcal{D}_{X}$-module via the natural left action of $\mathcal{D}_{X}$ on $\mathcal{O}_{X}$. As an example, we compute the pro-representing hull of $\mathcal{O}_{X}$ as a quasi-coherent left $\mathcal{D}_{X}$-module when $X$ is an elliptic curve, see also Eriksen [5].

Let $\mathrm{U}$ be an open affine cover of $X$. Then $U \subseteq X$ is a smooth, irreducible affine variety over $k$ of dimension $d$ for all $U \in \mathrm{U}$. It is well-known that $\mathcal{D}_{X}(U)$ is a simple Noetherian ring of global dimension $d$ and that $\mathcal{O}_{X}(U)$ is a simple left $\mathcal{D}_{X}(U)$-module, see Smith, Stafford [17]. Hence $\mathcal{E} x t_{\mathcal{D}_{X}}^{q}\left(\mathcal{O}_{X}, \mathcal{O}_{X}\right)=0$ for $q \geq d+1$ and $\mathcal{E} n d_{\mathcal{D}_{X}}\left(\mathcal{O}_{X}\right)=\underline{k}$. If $X$ is curve, then the spectral sequence in proposition 9 degenerates, and

$$
\begin{aligned}
& \mathrm{HH}^{n}\left(\mathrm{U}, \mathcal{D}_{X}, \mathcal{E} n d_{k}\left(\mathcal{O}_{X}\right)\right) \cong \mathrm{H}^{n-1}\left(\mathrm{U}, \mathcal{E} x t_{\mathcal{D}_{X}}^{1}\left(\mathcal{O}_{X}, \mathcal{O}_{X}\right)\right) \text { for } n \geq 1 \\
& \mathrm{HH}^{0}\left(\mathrm{U}, \mathcal{D}_{X}, \mathcal{E} n d_{k}\left(\mathcal{O}_{X}\right)\right) \cong k
\end{aligned}
$$

Let $X \subseteq \mathbf{P}^{2}$ be the irreducible projective plane curve given by the homogeneous equation $f=0$, where $f=y^{2} z-x^{3}-a x z^{2}-b z^{3}$ for fixed parameters $(a, b) \in k^{2}$. We assume that $\Delta=4 a^{3}+27 b^{2} \neq 0$, so that $X$ is smooth and therefore an elliptic curve over $k$. We choose an open affine cover $\mathrm{U}=\left\{U_{1}, U_{2}, U_{3}\right\}$ of $X$ closed under intersections, given by $U_{1}=D_{+}(y), U_{2}=D_{+}(z)$ and $U_{3}=U_{1} \cap U_{2}$. We shall compute $\mathcal{E} x t_{\mathcal{D}_{X}}^{1}\left(\mathcal{O}_{X}, \mathcal{O}_{X}\right)$ and $\mathrm{H}^{n-1}\left(\mathrm{U}, \mathcal{E} x t_{\mathcal{D}_{X}}^{1}\left(\mathcal{O}_{X}, \mathcal{O}_{X}\right)\right)$ for $n=1,2$.

Let $A_{i}=\mathcal{O}_{X}\left(U_{i}\right)$ and $D_{i}=\mathcal{D}_{X}\left(U_{i}\right)$ for $i=1,2,3$. We see that $A_{1} \cong k[x, z] /\left(f_{1}\right)$ and $A_{2} \cong k[x, y] /\left(f_{2}\right)$, where $f_{1}=z-x^{3}-a x z^{2}-b z^{3}$ and $f_{2}=y^{2}-x^{3}-a x-b$. Moreover, we have that $\operatorname{Der}_{k}\left(A_{i}\right)=A_{i} \partial_{i}$ and $D_{i}=A_{i}\left\langle\partial_{i}\right\rangle$ for $i=1,2$, where

$$
\begin{aligned}
& \partial_{1}=\left(1-2 a x z-3 b z^{2}\right) \partial / \partial x+\left(3 x^{2}+a z^{2}\right) \partial / \partial z \\
& \partial_{2}=-2 y \partial / \partial x-\left(3 x^{2}+a\right) \partial / \partial y
\end{aligned}
$$

On the intersection $U_{3}=U_{1} \cap U_{2}$, we choose an isomorphism $A_{3} \cong k\left[x, y, y^{-1}\right] /\left(f_{3}\right)$ with $f_{3}=f_{2}$, and see that $\operatorname{Der}_{k}\left(A_{3}\right)=A_{3} \partial_{3}$ and $D_{3}=A_{3}\left\langle\partial_{3}\right\rangle$ for $\partial_{3}=\partial_{2}$. The restriction maps of $\mathcal{O}_{X}$ and $\mathcal{D}_{X}$, considered as presheaves on $\mathrm{U}$, are given by

$$
x \mapsto x y^{-1}, z \mapsto y^{-1}, \partial_{1} \mapsto \partial_{2}
$$

for the inclusion $U_{1} \supseteq U_{3}$, and the natural localization map for $U_{2} \supseteq U_{3}$. Finally, we find a free resolution of $A_{i}$ as a left $D_{i}$-module for $i=1,2,3$, given by

$$
0 \leftarrow A_{i} \leftarrow D_{i} \stackrel{\cdot \partial_{i}}{\longleftarrow} D_{i} \leftarrow 0
$$

and use this to compute $\operatorname{Ext}_{D_{i}}^{1}\left(A_{i}, A_{j}\right) \cong \operatorname{coker}\left(\left.\partial_{i}\right|_{U_{j}}: A_{j} \rightarrow A_{j}\right)$ for all $U_{i} \supseteq U_{j}$ in $\mathrm{U}$. We see that $\operatorname{Ext}_{D_{i}}^{1}\left(A_{i}, A_{3}\right) \cong \operatorname{coker}\left(\partial_{3}: A_{3} \rightarrow A_{3}\right)$ is independent of $i$, and find the following $k$-linear bases for $\operatorname{Ext}_{D_{i}}^{1}\left(A_{i}, A_{j}\right)$ : 


\begin{tabular}{|l|l|l|}
\hline & $a \neq 0:$ & $a=0:$ \\
\hline$U_{1} \supseteq U_{1}$ & $1, z, z^{2}, z^{3}$ & $1, z, x, x z$ \\
$U_{2} \supseteq U_{2}$ & $1, y^{2}$ & $1, x$ \\
$U_{3} \supseteq U_{3}$ & $x^{2} y^{-1}, 1, y^{-1}, y^{-2}, y^{-3}$ & $x^{2} y^{-1}, 1, y^{-1}, x, x y^{-1}$ \\
\hline
\end{tabular}

The functor $\mathcal{E} x t_{\mathcal{D}_{X}}^{1}\left(\mathcal{O}_{X}, \mathcal{O}_{X}\right): \operatorname{Mor} \mathrm{U} \rightarrow \operatorname{Mod}(k)$ defines the following diagram in $\operatorname{Mod}(k)$, where the maps are induced by the restriction maps on $\mathcal{O}_{X}$ :

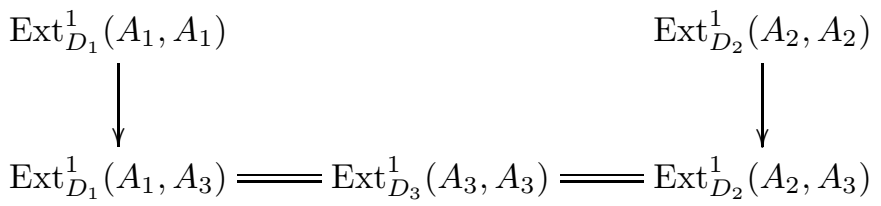

We use that $15 y^{2}=\Delta y^{-2}$ in $\operatorname{Ext}_{D_{3}}^{1}\left(A_{3}, A_{3}\right)$ when $a \neq 0$ and that $-3 b x y^{-2}=x$ in $\operatorname{Ext}_{D_{3}}^{1}\left(A_{3}, A_{3}\right)$ when $a=0$ to describe these maps in the given bases, and compute $\mathrm{H}^{n-1}\left(\mathrm{U}, \operatorname{Ext}_{\mathcal{D}_{X}}^{1}\left(\mathcal{O}_{X}, \mathcal{O}_{X}\right)\right)$ for $n=1,2$ using the resolving complex $\mathrm{D}^{*}(\mathrm{U},-)$. We find the following $k$-linear bases:

\begin{tabular}{|l|l|l|}
\hline & $a \neq 0:$ & $a=0:$ \\
\hline$n=1$ & $\xi_{1}=(1,1,1), \xi_{2}=\left(\Delta z^{2}, 15 y^{2}, \Delta y^{-2}\right)$ & $\xi_{1}=(1,1,1), \xi_{2}=(-3 b x z, x, x)$ \\
$n=2$ & $\omega=\left(0,0,0,0,6 a x^{2} y^{-1}\right)$ & $\omega=\left(0,0,0,0, x^{2} y^{-1}\right)$ \\
\hline
\end{tabular}

We recall that $\xi_{1}, \xi_{2}$ and $\omega$ are represented by cocycles of degree $p=0$ and $p=1$ in the resolving complex $\mathrm{D}^{*}\left(\mathrm{U}, \mathcal{E} x t_{\mathcal{D}_{X}}^{1}\left(\mathcal{O}_{X}, \mathcal{O}_{X}\right)\right)$, where

$$
\mathrm{D}^{p}\left(\mathrm{U}, \mathcal{E} x t_{\mathcal{D}_{X}}^{1}\left(\mathcal{O}_{X}, \mathcal{O}_{X}\right)\right)=\prod_{U_{0} \supseteq \cdots \supseteq U_{p}} \mathcal{E} x t_{\mathcal{D}_{X}}^{1}\left(\mathcal{O}_{X}, \mathcal{O}_{X}\right)\left(U_{0} \supseteq U_{p}\right)
$$

and the product is indexed by $\left\{U_{1} \supseteq U_{1}, U_{2} \supseteq U_{2}, U_{3} \supseteq U_{3}\right\}$ when $p=0$, and $\left\{U_{1} \supseteq U_{1}, U_{2} \supseteq U_{2}, U_{3} \supseteq U_{3}, U_{1} \supseteq U_{3}, U_{2} \supseteq U_{3}\right\}$ when $p=1$.

This proves that the noncommutative deformation functor $\operatorname{Def}_{\mathcal{O}_{X}}: a_{1} \rightarrow$ Sets of the left $\mathcal{D}_{X}$-module $\mathcal{O}_{X}$ has tangent space $\mathrm{HH}^{1}\left(\mathrm{U}, \mathcal{D}_{X}, \mathcal{E} n d_{k}\left(\mathcal{O}_{X}\right)\right) \cong k^{2}$ and obstruction space $\mathrm{HH}^{2}\left(\mathrm{U}, \mathcal{D}_{X}, \mathcal{E} n d_{k}\left(\mathcal{O}_{X}\right)\right) \cong k$ for any elliptic curve $X$ over $k$, and a pro-representing hull $H=k \ll t_{1}, t_{2} \gg /(F)$ for some noncommutative power series $F \in k \ll t_{1}, t_{2} \gg$.

We shall compute the noncommutative power series $F$ and the versal family $\mathcal{F}_{H} \in \operatorname{Def}_{\mathcal{O}_{X}}(H)$ using the obstruction calculus. We choose base vectors $t_{1}^{*}, t_{2}^{*}$ in $\mathrm{HH}^{1}\left(\mathrm{U}, \mathcal{A}, \mathcal{E} n d_{k}\left(\mathcal{O}_{X}\right)\right)$, and representatives $\left(\psi_{l}, \tau_{l}\right) \in \mathrm{D}^{01} \oplus \mathrm{D}^{10}$ of $t_{l}^{*}$ for $l=1,2$, where $\mathrm{D}^{p q}=\mathrm{D}^{p}\left(\mathrm{U}, \mathrm{HC}^{q}\left(\mathcal{A}, \mathcal{E} n d_{k}\left(\mathcal{O}_{X}\right)\right)\right)$. We may choose $\psi_{l}\left(U_{i}\right)$ to be the derivation defined by

$$
\psi_{l}\left(U_{i}\right)\left(P_{i}\right)= \begin{cases}0 & \text { if } P_{i} \in A_{i} \\ \xi_{l}\left(U_{i}\right) \cdot \operatorname{id}_{A_{i}} & \text { if } P_{i}=\partial_{i}\end{cases}
$$

for $l=1,2$ and $i=1,2,3$, and $\tau_{l}\left(U_{i} \supseteq U_{j}\right)$ to be the multiplication operator in $\operatorname{Hom}_{A_{i}}\left(A_{i}, A_{j}\right) \cong A_{j}$ given by $\tau_{1}=0, \tau_{2}\left(U_{i} \supseteq U_{i}\right)=0$ for $i=1,2,3$ and 


\begin{tabular}{|l|l|}
\hline$a \neq 0:$ & $a=0:$ \\
\hline$\tau_{2}\left(U_{1} \supseteq U_{3}\right)=0$ & $\tau_{2}\left(U_{1} \supseteq U_{3}\right)=x^{2} y^{-1}$ \\
$\tau_{2}\left(U_{2} \supseteq U_{3}\right)=-4 a^{2} y^{-1}-3 x y+9 b x y^{-1}-6 a x^{2} y^{-1}$ & $\tau_{2}\left(U_{2} \supseteq U_{3}\right)=0$ \\
\hline
\end{tabular}

The restriction of $\operatorname{Def}_{\mathcal{O}_{X}}: a_{1} \rightarrow$ Sets to $a_{1}(2)$ is represented by $\left(H_{2}, \mathcal{F}_{H_{2}}\right)$, where $H_{2}=k\left\langle t_{1}, t_{2}\right\rangle /\left(t_{1}, t_{2}\right)^{2}$ and the deformation $\mathcal{F}_{H_{2}} \in \operatorname{Def}_{\mathcal{O}_{X}}\left(H_{2}\right)$ is defined by $\mathcal{F}_{H_{2}}\left(U_{i}\right)=A_{i} \otimes_{k} H_{2}$ as a right $H_{2}$-module for $i=1,2,3$, with left $D_{i}$-module structure given by

$$
P_{i}\left(m_{i} \otimes 1\right)=P_{i}\left(m_{i}\right) \otimes 1+\psi_{1}\left(U_{i}\right)\left(P_{i}\right)\left(m_{i}\right) \otimes t_{1}+\psi_{2}\left(U_{i}\right)\left(P_{i}\right)\left(m_{i}\right) \otimes t_{2}
$$

for $i=1,2,3$ and for all $P_{i} \in D_{i}, m_{i} \in A_{i}$, and with restriction map for the inclusion $U_{i} \supseteq U_{j}$ given by

$$
\left.m_{i} \otimes 1 \mapsto m_{i}\right|_{U_{j}} \otimes 1+\left.\tau_{2}\left(U_{i} \supseteq U_{j}\right) m_{i}\right|_{U_{j}} \otimes t_{2}
$$

for $i=1,2, j=3$ and for all $m_{i} \in A_{i}$.

Let us attempt to lift the family $\mathcal{F}_{H_{2}} \in \operatorname{Def}_{\mathcal{O}_{X}}\left(H_{2}\right)$ to $R=k \ll t_{1}, t_{2} \gg /\left(t_{1}, t_{2}\right)^{3}$. We let $\mathcal{F}_{R}\left(U_{i}\right)=A_{i} \otimes_{k} R$ as a right $R$-module for $i=1,2,3$, with left $D_{i}$-module structure given by

$$
P_{i}\left(m_{i} \otimes 1\right)=P_{i}\left(m_{i}\right) \otimes 1+\psi_{1}\left(U_{i}\right)\left(P_{i}\right)\left(m_{i}\right) \otimes t_{1}+\psi_{2}\left(U_{i}\right)\left(P_{i}\right)\left(m_{i}\right) \otimes t_{2}
$$

for $i=1,2,3$ and for all $P_{i} \in D_{i}, m_{i} \in A_{i}$, and with restriction map for the inclusion $U_{i} \supseteq U_{j}$ given by

$$
\left.m_{i} \otimes 1 \mapsto m_{i}\right|_{U_{j}} \otimes 1+\left.\tau_{2}\left(U_{i} \supseteq U_{j}\right) m_{i}\right|_{U_{j}} \otimes t_{2}+\left.\frac{\tau_{2}\left(U_{i} \supseteq U_{j}\right)^{2}}{2} m_{i}\right|_{U_{j}} \otimes t_{2}^{2}
$$

for $i=1,2, j=3$ and for all $m_{i} \in A_{i}$. We see that $\mathcal{F}_{R}\left(U_{i}\right)$ is a left $\mathcal{D}_{X}\left(U_{i}\right)$-module for $i=1,2,3$, and that $t_{1} t_{2}-t_{2} t_{1}=0$ is a necessary and sufficient condition for $\mathcal{D}_{X}$-linearity of the restriction maps for the inclusions $U_{1} \supseteq U_{3}$ and $U_{2} \supseteq U_{3}$. This implies that $\mathcal{F}_{R}$ is not a lifting of $\mathcal{F}_{H_{2}}$ to $R$. But if we consider the quotient $H_{3}=R /\left(t_{1} t_{2}-t_{2} t_{1}\right)$, we see that the family $\mathcal{F}_{H_{3}} \in \operatorname{Def}_{\mathcal{O}_{X}}\left(H_{3}\right)$ induced by $\mathcal{F}_{R}$ is a lifting of $\mathcal{F}_{\mathrm{H}_{2}}$ to $\mathrm{H}_{3}$.

In fact, we claim that the restriction of $\operatorname{Def}_{\mathcal{O}_{X}}: a_{1} \rightarrow$ Sets to $a_{1}(3)$ is represented by $\left(H_{3}, \mathcal{F}_{H_{3}}\right)$. One way to prove this is to show that it is not possible to find any lifting $\mathcal{F}_{R}^{\prime} \in \operatorname{Def}_{\mathcal{O}_{X}}(R)$ of $\mathcal{F}_{H_{2}}$ to $R$. Another approach is to calculate the cup products $<t_{i}^{*}, t_{j}^{*}>$ in global Hochschild cohomology for $i, j=1,2$, and this gives

$$
\begin{array}{ll}
<t_{1}^{*}, t_{2}^{*}>=o^{*},<t_{2}^{*}, t_{1}^{*}>=-o^{*} & \text { for } a \neq 0 \\
<t_{1}^{*}, t_{2}^{*}>=o^{*},<t_{2}^{*}, t_{1}^{*}>=-o^{*} & \text { for } a=0
\end{array}
$$

where $o^{*} \in \mathrm{HH}^{2}\left(\mathrm{U}, \mathcal{D}_{X}, \mathcal{E} n d_{k}\left(\mathcal{O}_{X}\right)\right)$ is the base vector corresponding to $\omega$. Since all other cup products vanish, this implies that $F=t_{1} t_{2}-t_{2} t_{1} \bmod \left(t_{1}, t_{2}\right)^{3}$.

Let $H=k \ll t_{1}, t_{2} \gg /\left(t_{1} t_{2}-t_{2} t_{1}\right)$. We shall show that it is possible to find a lifting $\mathcal{F}_{H} \in \operatorname{Def}_{\mathcal{O}_{X}}(H)$ of $\mathcal{F}_{H_{3}}$ to $H$. We let $\mathcal{F}_{\mathbf{H}}\left(U_{i}\right)=A_{i} \widehat{\otimes}_{k} H$ as a right $H$-module for $i=1,2,3$, with left $D_{i}$-module structure given by

$$
P_{i}\left(m_{i} \otimes 1\right)=P_{i}\left(m_{i}\right) \otimes 1+\psi_{1}\left(U_{i}\right)\left(P_{i}\right)\left(m_{i}\right) \otimes t_{1}+\psi_{2}\left(U_{i}\right)\left(P_{i}\right)\left(m_{i}\right) \otimes t_{2}
$$

for $i=1,2,3$ and for all $P_{i} \in \mathcal{D}_{i}, m_{i} \in A_{i}$, and with restriction map for the inclusion $U_{i} \supseteq U_{j}$ given by

$$
\left.m_{i} \otimes 1 \mapsto \sum_{n=0}^{\infty} \frac{\tau_{2}\left(U_{i} \supseteq U_{j}\right)^{n}}{n !} m_{1}\right|_{U_{j}} \otimes t_{2}^{n}=\exp \left(\tau_{2}\left(U_{i} \supseteq U_{j}\right) \otimes t_{2}\right) \cdot\left(\left.m_{1}\right|_{U_{j}} \otimes 1\right)
$$


for $i=1,2, j=3$ and for all $m_{i} \in A_{i}$. This implies that $\left(H, \mathcal{F}_{H}\right)$ is the prorepresenting hull of $\operatorname{Def}_{\mathcal{O}_{X}}$, and that $F=t_{1} t_{2}-t_{2} t_{1}$. We remark that the versal family $\mathcal{F}_{H}$ does not admit an algebraization, i.e. an algebra $H_{\text {alg }}$ of finite type over $k$ such that $H$ is a completion of $H_{\text {alg }}$, together with a deformation in $\operatorname{Def}_{\mathcal{O}_{X}}\left(H_{\text {alg }}\right)$ that induces the versal family $\mathcal{F}_{H} \in \operatorname{Def}_{\mathcal{O}_{X}}(H)$.

\section{REFERENCES}

1. A. Beı̆linson and J. Bernstein, A proof of Jantzen conjectures, I. M. Gel'fand Seminar, Adv. Soviet Math., vol. 16, Amer. Math. Soc., Providence, RI, 1993, pp. 1-50. MR MR1237825 (95a:22022)

2. Alexandre Beulinson and Joseph Bernstein, Localisation de g-modules, C. R. Acad. Sci. Paris Sér. I Math. 292 (1981), no. 1, 15-18. MR MR610137 (82k:14015)

3. N. Bourbaki, Éléments de mathématique. Fascicule XXVII. Algèbre commutative. Chapitre 1: Modules plats. Chapitre 2: Localisation, Actualités Scientifiques et Industrielles, No. 1290, Herman, Paris, 1961. MR MR0217051 (36 \#146)

4. Eivind Eriksen, Iterated extensions in module categories, arXiv: math/0406034 v1, 2004.

5. _ Computing noncommutative global deformations of $\mathcal{D}$-modules, arXiv: math/0612441 v2, 2006.

6. A. Grothendieck, Éléments de géométrie algébrique. I. Le langage des schémas, Inst. Hautes Études Sci. Publ. Math. (1960), no. 4, 228. MR MR0217083 (36 \#177a)

7. É Éléments de géométrie algébrique. III. Étude cohomologique des faisceaux cohérents. I, Inst. Hautes Études Sci. Publ. Math. (1961), no. 11, 167. MR MR0217085 (36 \#177c)

8. _ Éléments de géométrie algébrique. IV. Étude locale des schémas et des morphismes de schémas IV, Inst. Hautes Études Sci. Publ. Math. (1967), no. 32, 361. MR MR0238860 (39 \#220)

9. Alexander Grothendieck, Fondements de la géométrie algébrique. [Extraits du Séminaire Bourbaki, 1957-1962.], Secrétariat mathématique, Paris, 1962. MR MR0146040 (26 \#3566)

10. - Géométrie formelle et géométrie algébrique, Séminaire Bourbaki, Vol. 5, Soc. Math. France, Paris, 1995, pp. Exp. No. 182, 193-220, errata p. 390. MR MR1603467

11. — Technique de descente et théorèmes d'existence en géometrie algébrique. I. Généralités. Descente par morphismes fidèlement plats, Séminaire Bourbaki, Vol. 5, Soc. Math. France, Paris, 1995, pp. Exp. No. 190, 299-327. MR MR1603475

12. Robin Hartshorne, Algebraic geometry, Springer-Verlag, New York, 1977, Graduate Texts in Mathematics, No. 52. MR MR0463157 (57 \#3116)

13. O. A. Laudal, Matric Massey products and formal moduli. I, Algebra, algebraic topology and their interactions (Stockholm, 1983), Lecture Notes in Math., vol. 1183, Springer, Berlin, 1986, pp. 218-240. MR MR846451 (87k:55023)

14. Noncommutative deformations of modules, Homology Homotopy Appl. 4 (2002), no. 2, part 2, 357-396 (electronic), The Roos Festschrift volume, 2. MR MR1918517 (2003e:16005)

15. Olav Arnfinn Laudal, Formal moduli of algebraic structures, Lecture Notes in Mathematics, vol. 754, Springer, Berlin, 1979. MR MR551624 (82h:14009)

16. Michael Schlessinger, Functors of Artin rings, Trans. Amer. Math. Soc. 130 (1968), 208-222. MR MR0217093 (36 \#184)

17. S. P. Smith and J. T. Stafford, Differential operators on an affine curve, Proc. London Math. Soc. (3) 56 (1988), no. 2, 229-259. MR MR922654 (89d:14039)

18. Michel Van den Bergh, Differential operators on semi-invariants for tori and weighted projective spaces, Topics in invariant theory (Paris, 1989/1990), Lecture Notes in Math., vol. 1478, Springer, Berlin, 1991, pp. 255-272. MR MR1180993 (93h:16046)

19. Amnon Yekutieli and James J. Zhang, Dualizing complexes and perverse modules over differential algebras, Compos. Math. 141 (2005), no. 3, 620-654. MR MR2135281 (2006c:16014)

Oslo University College, Postboks 4 St. Olavs Plass, 0130 Oslo, Norway

E-mail address: eeriksen@hio.no 